\title{
Magnetic metal oxide-organic framework material for ultrasonic-assisted sorption of titan yellow and rose bengal from aqueous solutions
}

\author{
Khalid Z. Elwakeel $^{\mathrm{a}, \mathrm{b}}$, Ahmed Shahat ${ }^{\mathrm{c}}$, Ziya A. Khan ${ }^{\mathrm{a}}$, Wael Alshitari ${ }^{\mathrm{a}}$, Eric Guibal ${ }^{\mathrm{d}, *}$ \\ ${ }^{\text {a }}$ University of Jeddah, College of Science, Department of Chemistry, Jeddah, Saudi Arabia \\ ${ }^{\mathrm{b}}$ Environmental Science Department, Faculty of Science, Port-Said University, Port-Said, Egypt \\ ${ }^{\mathrm{c}}$ Department of Chemistry, Faculty of Science, Suez University, Suez, Egypt \\ ${ }^{\mathrm{d}}$ IMT - Mines Ales, Polymers, Composites \& Hybrides (PCH), Alès, France
}

Thiourea-formaldehyde (TF) resin is prepared by polycondensation of its two precursors; the incorporation of pre-formed magnetite particles allows synthesizing a magnetic derivative of TF (MTF). These materials are applied to the sorption of two dyes (titan yellow, TY, and rose bengal, RB). Ultrasonic treatment (UT) is tested as an alternative to mechanical agitation (MA). The two sorbents are characterized by scanning electron microscopy, vibrating-sample magnetometry, BET surface area analysis, thermogravimetric analysis, Fourier-transform infrared and NMR spectroscopy, zetametry. Sorption properties are compared for TF and MTF under both MA and UT; considering pH effect, uptake kinetics (modeled using the pseudo-second order rate equation, and resistance to intraparticle diffusion), sorption isotherms (fitted by the Langmuir and Sips equations), the effect of competitor ions, and the desorption efficiency. Optimum sorption occurs at $\mathrm{pH} 3$ (balance between (a) the electrostatic interactions between protonated groups (sorbent) and anionic forms of the dyes, and (b) the competitor effects of counter anions). UT strongly decreases the contact time required for reaching equilibrium (from $240 \mathrm{~min}$ to $30 \mathrm{~min}$ ). UT increases the sorption capacities: in the range $0.3-0.6 \mathrm{mmol} \mathrm{dye}^{-1}$ (MA) up to 0.9-1 mmol dye $\mathrm{g}^{-1}$. The magnetic core does not change kinetics, while it increases TF sorption capacities for both TY and RB. Under UT, the maximum sorption capacities are comparable for TF and MTF; however, the affinity of MTF remains higher than TF for the two dyes. Alkaline NaCl solutions completely desorb the dyes; UT decreases both the contact time and the $\mathrm{NaCl}$ concentration required for achieving the complete desorption of loaded dyes. The sorbents are recycled with high stability in sorption performance for at least five cycles.

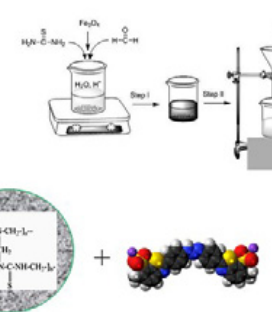

TF/MTF sorbent

Dye molecule
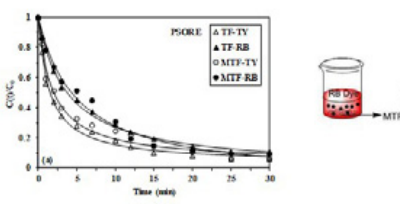

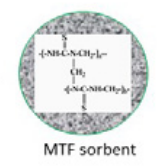

MTF sorbent
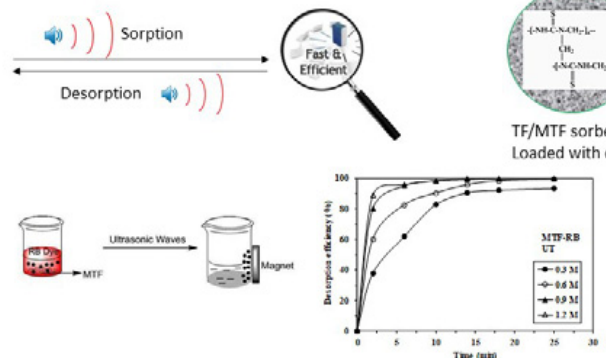

Keywords:

Dyes

Magnetic thiourea-formaldehyde resin

Ultrasonic treatment

Sorption isotherms

Uptake kinetics

Desorption

\footnotetext{
* Corresponding author.

E-mail address: Eric.Guibal@mines-ales.fr (E. Guibal).
} 


\section{Introduction}

The regulations at the World level became drastically more stringent for the last decades under the pressure of climate changes and expectative of populations for more environmentally friendly management of resources (including water). Therefore, industrial activities are strongly controlled in terms of water discharge into the environment. In this context, a great attention is paid to wastewater treatment for decreasing the concentration of noxious contaminants such as organic dyes, heavy metal ions, microbes, and pharmaceuticals that can cause serious damages to biotope $[1,2]$. In particular, the behavior of dyes in the environment is of critical importance because of their direct impact on photosynthesis for ecosystem and on human health, and because of their high resilience in conventional water treatments $[3,4]$. Indeed, many organic dyes are resistant against physical, chemical and biological treatments $[5,6]$. The high solubility of dyes, combined with the specific activity of enzymes and bacteria from the intestinal microflora, can induce the formation of aromatic amines, which are highly toxic, mutagenic and carcinogenic $[7,8]$.

The classical methods, such as coagulation and flocculation [9], electrocoagulation [10] may face important limitations (incomplete removal) or drawbacks such as the release of huge amounts of contaminated sludge [11]. Therefore, the development of alternative energy-efficient processes for the removal of dye contaminants is necessary. Different kinds of advanced technologies have been developed such as catalytic photodegradation [12], or nanofiltration [13]; these technologies may have some drawbacks such as generating some very hazardous sub-products or contaminated concentrates, respectively. Sorption processes constitute an interesting alternative [4,14]; their high efficiency, easy operability may explain that they are frequently recommended for the treatment of low-concentration effluents or as polishing treatment. Many materials have been reported for their performance for dye recovery using activated carbon [15], silica [16], biosorbents [17-19]. Recently, metal oxides and metal-organic frameworks (MOF) have been investigated for dye recovery [20,21]; they show high sorption capacity and low cost. Several kinds of metal oxides have been investigated such as $\mathrm{MnO}_{2}$ [22,23], $\mathrm{Fe}_{2} \mathrm{O}_{3}$ [24], $\mathrm{TiO}_{2}$ [25], and $\mathrm{MgO}$ [26], ionic liquid-modified $\mathrm{Fe}_{3} \mathrm{O}_{4}$ [27], and activated carbonmodified $\mathrm{Fe}_{3} \mathrm{O}_{4}$ [28]. Magnetic-based materials have been recognized as promising sorbents because of their large surface areas [29] and small diffusion resistance [29]. Indeed, the possibility to recover magnetically magnetite-based sorbents allows designing small-size particles with limited control of resistance to intraparticle diffusion. Hybrid metal-organic frameworks offer multifunctional properties with highly porous characteristics [29-31].

The polycondensation of thiourea and formaldehyde allows preparing resins (TF) with high affinity for the sorption of various metals ions [30,32-37]. To date, it seems that TF resins have not been used for the recovery of dyes and more generally organic contaminants. This study investigates the sorption properties of two dyes (titan yellow, TY, and rose bengal, $\mathrm{RB}$ ) on two TF resins: the raw TF resin and another sorbent prepared by embedment of magnetite nanoparticles (MTF). The effect of magnetite on porous characteristics and indirect influence on sorption properties are explored. A special attention is paid to uptake kinetics and to the mechanisms of resistance to intraparticle diffusion. A first part of this study focuses on the characterization of the two materials using a wide range of analytical techniques: scanning electron microscopy (SEM), transmission electron microscopy (TEM), textural analysis (BET), dynamic light scattering (DLS), vibrating-sample magnetometry (VSM), FTIR spectroscopy, ${ }^{1} \mathrm{H}$ and ${ }^{13} \mathrm{C}$ NMR spectroscopies, zetametry.

The enhancement of uptake kinetics is a challenge for sorption processes. Several strategies have been investigated: decreasing the size of sorbent particles (magnetite incorporation facilitates solid/liquid separation at the end of the process), expanding the structure of the material, or changing the textural properties (specific surface area, porosity). The mode of agitation is also of critical importance; ultrasound treatment allows increasing the leaching efficiency in hydrometallurgy $[38,39]$ but also the sorption performances $[28,40-44]$. The cavitation effect allows increasing the accessibility to internal porous network: ultrasonic treatment was used for incorporating ionic liquid into chitosan (for preparing sorbents with enhanced properties for $\mathrm{Cs}(\mathrm{I})$ and Sr(II) removal) [45].

In order to evaluate the cross-effects of magnetic particles (MTF $v s$. TF) and agitation mode (mechanical stirring $v s$. ultrasonic treatment), the sorption properties of the two resins for two anionic dyes are investigated: titan yellow (TY) and rose bengal (RB) that bear sulfonic and carboxylic acid groups, respectively (Scheme AM1, see Additional Material Section, AMS). The study investigates the influence of different experimental conditions on sorption properties; including $\mathrm{pH}$, initial dye concentration, uptake kinetics, and the composition of the solution (presence of competitor anions). Sorption isotherms are carried out and some tests performed at different temperatures allow determining the thermodynamic parameters. Finally, the desorption of loaded dyes from the sorbents is investigated using alkaline $\mathrm{NaCl}$ solutions.

\section{Materials and methods}

\subsection{Materials}

Thiourea, formaldehyde solution $(37 \% \mathrm{w} / \mathrm{w})$, iron(III) chloride $\left(\mathrm{FeCl}_{3} \cdot 6 \mathrm{H}_{2} \mathrm{O}\right)$ and iron(II) sulfate $\left(\mathrm{FeSO}_{4} \cdot 7 \mathrm{H}_{2} \mathrm{O}\right)$ were provided by Sigma-Aldrich (Merck, Darmastadt, Germany), as analytical grade reagents. Titan Yellow (TY, Thiazole Yellow G) was supplied by Loba Chemie (Mumbai, India), while Rose Bengal (RB) was purchased from Fluka (Buchs, Switzerland) (Scheme AM1, see Additional Material Section, AMS). NaCl, $\mathrm{Na}_{3} \mathrm{PO}_{4} \cdot 12 \mathrm{H}_{2} \mathrm{O}$ and $\mathrm{Na}_{2} \mathrm{SO}_{4}$ and $\mathrm{NaNO}_{3}$ were purchased from Sigma-Aldrich (Darmstadt, Germany)

\subsection{Synthesis of materials}

\subsubsection{Synthesis of magnetite particles}

The synthesis of magnetite nanoparticles was already described [46]. Briefly, magnetite particles were synthesized by the hydrothermal co-precipitation of ferric and ferrous salts [47]. Firstly, $\mathrm{FeSO}_{4} \cdot 7 \mathrm{H}_{2} \mathrm{O}$ $(3.334 \mathrm{~g})$ and $\mathrm{FeCl}_{3} \cdot 6 \mathrm{H}_{2} \mathrm{O}(6.480 \mathrm{~g})$ were dissolved in $150 \mathrm{~mL}$ of distilled water. The as-dissolved iron mixture was heated under reflux $(333 \pm 1 \mathrm{~K})$ in a 250 -mL flask. The mixture was magnetically stirred for 60 min under $\mathrm{N}_{2}$ atmosphere. Then, $\mathrm{NaOH}$ ( $3 \mathrm{M}$ ) solution was slowly and progressively added to the mixture until $\mathrm{pH}$ reached 12 ; the reaction was performed at the temperature of $318 \mathrm{~K}$ for $5 \mathrm{~h}$. The magnetite particles were magnetically separated and washed repeatedly with Milli-Q water (Millipore, Billerica, MA) until the suspension reached a $\mathrm{pH}$ close to 7 . Finally, the solid was rinsed with acetone, and dried, under vacuum, at $333 \pm 1 \mathrm{~K}$, for $5 \mathrm{~h}$.

\subsubsection{Synthesis of TF and MTF sorbents [32]}

A fixed amount of thiourea $(15.2 \mathrm{~g}, 0.2 \mathrm{~mol})$ was dissolved in $40 \mathrm{~mL}$ of demineralized water in a $250 \mathrm{~mL}$ round flask equipped with a stirrer and a condenser. The mixture was heated under agitation until complete dissolving of thiourea salt. In the next step, formaldehyde $(15 \mathrm{~mL}$ of $37 \% \mathrm{w} / \mathrm{w}$ aqueous solution, $0.2 \mathrm{~mol}$ ) was added to the solution. The $\mathrm{pH}$ was controlled by addition of $2 \mathrm{~mL}$ of glacial acetic acid (99.1\%). The mixture was maintained under agitation at $368 \pm 2 \mathrm{~K}$ for $4 \mathrm{~h}$. The white product (TF) was recovered by filtration and carefully washed for removing unreacted reagents and finally dried under vacuum for $6 \mathrm{~h}$ at $323 \pm 1 \mathrm{~K}$. The synthesis was almost quantitative:the yield was close to $97 \%$; i.e., $20.33 \mathrm{~g}$ of product for a total of $21 \mathrm{~g}$ of reagents.

For the synthesis of magnetic TF (MTF) the same procedure was applied (Scheme 1); the only variant consisted of incorporating $7.6 \mathrm{~g}$ of magnetite particles in the initial mixture of thiourea and formaldehyde (before increasing the temperature); all other processing parameters 


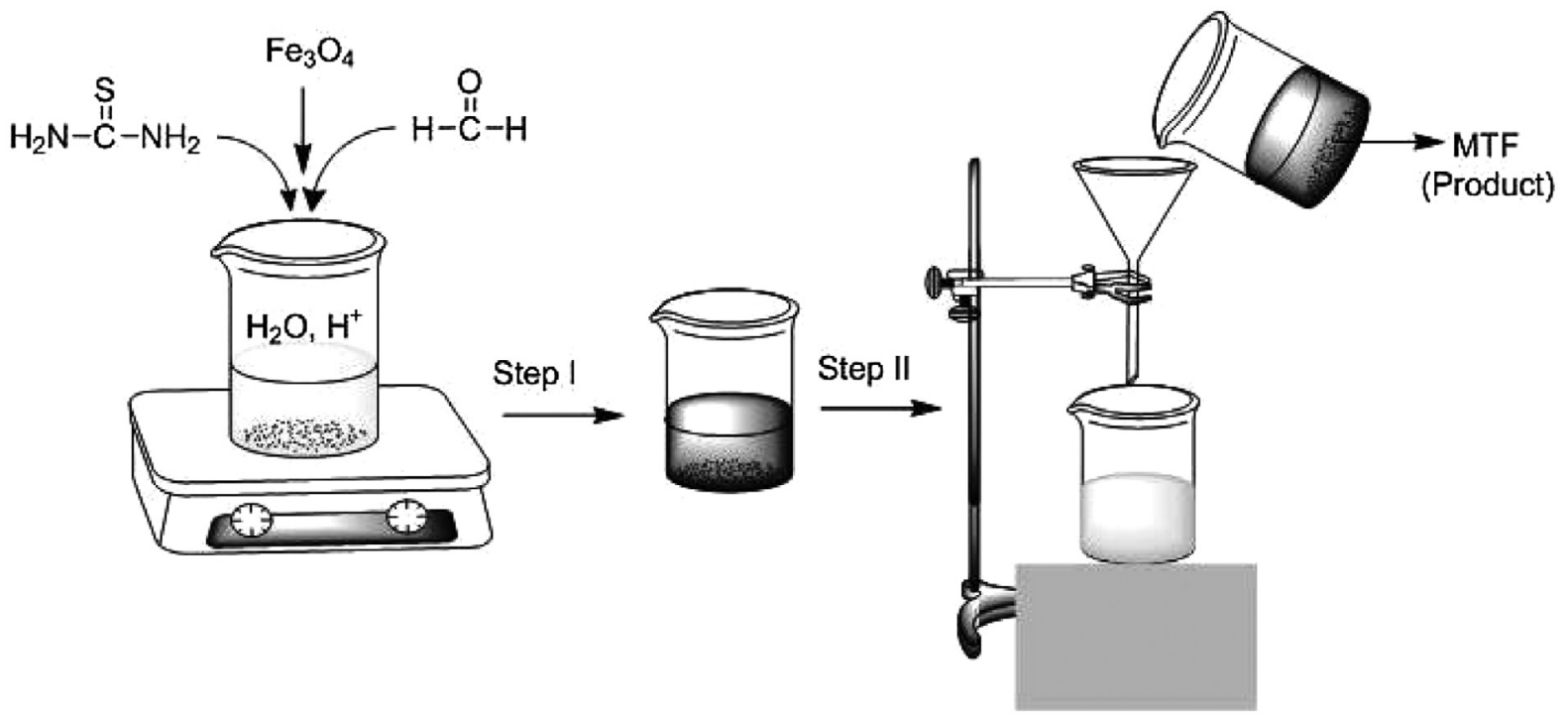

Scheme 1. Schematic diagram of MTF sorbent manufacturing.

being maintained. The composite material was abundantly rinsed with water under strong agitation and the solid was recovered by magnetic separation in order to remove any organic fraction that could be weakly bound to the magnetic nanoparticles.

\subsection{Characterization of materials}

The Fourier-Transform infrared spectra were obtained using a Nicolet IS10 FTIR (Thermo Fisher Scientific, Waltham, MA, USA) equipped with an ATR accessory (attenuated total reflectance). The structure of TF resin was also characterized using ${ }^{1} \mathrm{H}$ and ${ }^{13} \mathrm{C}$ NMR spectroscopies (on a BrukerAvance-III $400 \mathrm{MHz}$, Bern, Switzerland); polymer sample was prepared in deuterated DMSO solvent prior to analysis. The surface area determination and pore analysis of the sorbent beads (BET surface, pore volume and pore size) were performed using a Quantachrome NOVA 3200e (Quantachrome Instruments, Boynton Beach, FL, USA); and the data were analyzed using NovaWin software (v11.0). Thermogravimetric analysis was operated on Shimadzu TGA-50 thermogravimetric analyzer (Shimadzu Corporation, Tokyo, Japan). The surface charge measurements of the sorbent (zeta potential) were performed using a Nano Zeta Sizer (Nano-ZS Malvern Instruments Ltd., London, United Kingdom) at various $\mathrm{pH}$ values (from 1.0 to 8.0 ). The sorbent particles $(0.01 \mathrm{~g})$ were suspended in $50 \mathrm{~mL}$ of $0.1 \mathrm{M} \mathrm{KCl}$ solution for $2 \mathrm{~h}$ before analysis.

Magnetization tests were carried out at room temperature using a vibrating sample magnetometer (VSM: Princeton Measurements Corporation, PMC MicroMag 3900 model, Princeton, NJ, USA), applying a maximum magnetic field of $10 \mathrm{kOe}$. The morphology of the sorbent and the semi-quantitative chemical analysis of the sorbent before and after dye binding were obtained using a scanning electron microscope equipped with an integrated energy dispersive x-ray analyzer (JSM-6510LV, Jeol, Tokyo, Japan). Transmission electron microscope (TEM-2100HR, JEOL, Tokyo, Japan) was used for the ultrahigh resolution analysis of magnetite nanoparticles, the particles were suspended in demineralized water before being spread on carbon grids. The size of magnetite nanoparticles and sorbent microparticles (TF and MTF) was analyzed by dynamic light scattering using a DLS particle size analyzer NanoBrook 90Plus (Brookhaven Instruments Corporation, Holtsville, NY, USA).

The stability of sorbents in different solvents (such as methanol, ethanol, acetone, strong acid and bases, such as $2 \mathrm{M} \mathrm{HCl}, 2 \mathrm{M} \mathrm{NaOH}$, respectively) was evaluated under ultrasonic treatment. A definite weight of the sorbent was sonicated with each of the previous solvents for $3 \mathrm{~h}$, before being filtered and dried at $333 \pm 1 \mathrm{~K}$. Based on the mass difference (before and after sonication) it was possible calculating the percentage of sorbent dissolved or degraded. An ultrasonic bath sonicator (Sonorex Digitec DT 1028F, Bandelin electronic GmbH \& Co, KG, Berlin Germany), equipped with a shaking device (DA 1028H, Bandelin electronic $\mathrm{GmbH} \& \mathrm{Co}$ ), was used.

\subsection{Sorption experiments}

Dye solutions were prepared by dilution of the stock solution with ultrapure water (Milli-Q), just prior experiments. The impact of the $\mathrm{pH}$ on dye uptake was investigated by contact of $50 \mathrm{~mL}(\mathrm{~V})$ of dye solution $\left(\mathrm{C}_{0}: 1.0 \mathrm{mmol} \mathrm{L}^{-1}\right)$ with $0.05 \mathrm{~g}$ of sorbent $(\mathrm{m}$, dry weight). Sorption experiments were performed using a shaking incubator (LSI-3016R, Labtech, Sorisole (BG), Italy) at room temperature (i.e., $298 \pm 1 \mathrm{~K}$ ), unless specified. The value of initial $\mathrm{pH}$ was varied between 1 and 10 using $\mathrm{HCl}$ and $\mathrm{NaOH}(0.0001-0.01 \mathrm{M})$. The $\mathrm{pH}$ before and after sorption were measured using a pH meter (HANNA 211, Hanna Instruments, Lingolsheim, France). The suspension was stirred under an agitation speed of $210 \mathrm{rpm}$ for $180 \mathrm{~min}$; indeed, preliminary experiments showed that equilibrium is reached within $180 \mathrm{~min}$. After contact, the sorbents were separated from the solutions by filtration for TF using filter membranes (Whatmann, Merck, Kenilworth, NJ, USA) or by magnetic separation for MTF. The filtrates were analyzed for residual dye concentration using UV-Visible spectrophotometer at the proper wavelengths ( $400 \mathrm{~nm}$ for TY and $545 \mathrm{~nm}$ for RB). Analytical conditions were adjusted to fixed $\mathrm{pH}$ value for the samples to prevent any shift in the wavelength of the dyes. The concentration of dye solution was determined using U/VIS spectrophotometer (T70 + UV/VIS spectrophotometer, PG instruments Ltd, UK). Experiments were systematically duplicated; when the standard deviation exceeded $10 \%$, the experiment was triplicated (see Additional Material Section for examples of reproducibility tests).

Sorption isotherms were obtained by contact of $0.05 \mathrm{~g}$ of sorbent (m) with a fixed volume of solution $(\mathrm{V}=50 \mathrm{~mL})$ of dye at different initial concentrations $\left(\mathrm{C}_{0}\right.$, ranging between 0.1 and $\left.1.0 \mathrm{mmol} \mathrm{L}^{-1}\right)$ for $180 \mathrm{~min}$. The $\mathrm{pH}$ of the solutions was initially set at the optimum $\mathrm{pH}$, after solid/liquid separation, the equilibrium $\mathrm{pH}$ and the residual dye concentration $\left(\mathrm{C}_{\mathrm{eq}}, \mathrm{mmol}\right.$ dye $\left.\mathrm{L}^{-1}\right)$ were measured; the amount of sorbed dye $\left(\mathrm{q}_{\mathrm{e}}, \mathrm{mmol} \mathrm{g}^{-1}\right)$ was estimated by the mass balance equation:

$q_{e q}=\frac{\left(C_{0}-C_{e q}\right) V}{m}$ 
For uptake kinetics, a fixed amount of sorbent was mixed with a fixed volume of dye solution (sorbent dosage, SD: $1 \mathrm{~g} \mathrm{~L}^{-1}$ ) at $\mathrm{pH}$ 3.0. Samples $(4 \mathrm{~mL}$ ) were collected (by filtration or magnetic separation for $\mathrm{TF}$ and MTF, respectively) at fixed time intervals, then the residual concentrations of dye were determined by UV-VIS spectrophotometry. The agitation speed was set at $210 \mathrm{rpm}$, while the temperature was maintained at $298 \pm 1 \mathrm{~K}$. The sorbed amount of dye per unit weight of the sorbent at time $\mathrm{t}\left(\mathrm{q}_{(\mathrm{t})}\right.$, mmol dye $\left.\mathrm{g}^{-1}\right)$ was calculated from the mass balance equation (taking into account the decrement in the volume of the solution).

Sorption experiments were performed in shaking incubator (LSI$3016 \mathrm{R})$ at different temperatures $(298 \mathrm{~K}, 308 \mathrm{~K}, 318 \mathrm{~K}$ and $328 \mathrm{~K}$; temperature variation did not exceed $1 \mathrm{~K}$, compared to set value). After solid/liquid separation, the residual concentrations $\left(\mathrm{C}_{\mathrm{eq}}, \mathrm{mol} \mathrm{L}^{-1}\right)$ were determined and the sorption capacities $\left(\mathrm{q}_{\mathrm{eq}}, \mathrm{mmol} \mathrm{g}^{-1}\right)$ were calculated using the mass balance Eq. (1). The distribution ratio, $\mathrm{D}\left(\mathrm{L} \mathrm{g}^{-1}\right)$ was calculated according: $\mathrm{D}=\mathrm{q}_{\mathrm{eq}} / \mathrm{C}_{\mathrm{eq}}$. The influence of competitor or interfering anions such as chloride, nitrate, phosphate and sulfate, was also studied by preparing $1 \mathrm{mmol} \mathrm{L}^{-1}$ solutions of dyes diluted in solutions containing increasing amounts of $\mathrm{NaCl}, \mathrm{Na}_{3} \mathrm{PO}_{4} \cdot 12 \mathrm{H}_{2} \mathrm{O}$ and $\mathrm{Na}_{2} \mathrm{SO}_{4}$ and $\mathrm{NaNO}_{3}$. The range of initial concentration of competitor anions was $0.2-1.8 \mathrm{~mol} \mathrm{~L}^{-1}$ and the $\mathrm{pH}$ of the solutions was set to 3.2 . The sorbent dosage was fixed to $1 \mathrm{~g} \mathrm{~L}^{-1}$.

Sono-sorption was performed by contact of twenty $\mathrm{mL}$ of $1.0 \mathrm{mmol} \mathrm{L}^{-1}$ of TY and RB solutions at the optimum $\mathrm{pH}$ (deduced from sorption tests under mechanical agitation) with $0.05 \mathrm{~g}$ of sorbents (TF and MTF). The sorption process was carried out under ultrasound at the frequency of $35 \mathrm{kHz}$. In order to investigate the influence of ultrasonic experiments on the sorption kinetics and isotherms of TY and RB, the sorption experiments were conducted under the same conditions (described above), the unique difference consisted of using an ultrasonic bath sonicator (frequency: $35 \mathrm{kHz}$; Power: $300 \mathrm{~W}$ ) instead of the shaking incubator, at controlled temperature in a thermostatic box. After sono-sorption experiments, samples were withdrawn and immediately centrifuged, filtered or magnetically separated. The residual concentrations of TY and RB were determined by UV-VIS spectrophotometry. Blank tests (without sorbent) were initially carried out for verifying that the ultrasonic treatment does not affect the stability of the dye.

Conventional equations were used for modeling uptake kinetics and sorption isotherms. Tables AM1 and AM2 report the pseudo-first order rate equation (PFORE), the pseudo-second order rate equation (PSORE) and the resistance to intraparticle diffusion (RIDE, Crank equation) for uptake kinetics and the Langmuir, Freundlich, Sips and Temkin equations for sorption isotherms. The facilities of Mathematica ${ }^{\circledR}$ were systematically used for the non-linear regression and determination of model parameters.

Note: full experimental conditions are systematically reported in the caption of the figures and tables.

\subsection{Desorption experiments}

After being loaded with dyes under optimum conditions, the magnetic sorbent (MTF) was separated magnetically while the non-magnetic sorbent (TF) was recovered from the liquid phase by filtration. The sorbents were then washed with ultrapure water and dried. The mass balance equation was used to quantify the effective amount of dyes immobilized on the materials. A fixed amount of sorbent was mixed with a fixed volume of alkaline $\mathrm{NaCl}$ solutions (SD: $2.5 \mathrm{~g} \mathrm{~L}^{-1}$ ) with increasing concentrations $\left(0.2-1.8 \mathrm{~mol} \mathrm{~L}^{-1}\right)(\mathrm{pH} \mathrm{12)}$. Samples were collected at fixed times for evaluating the desorbed amounts of dyes and plotting the desorption kinetics. The desorption process was performed in shaking incubator at $210 \mathrm{rpm}$ and under ultrasound at the frequency of $35 \mathrm{kHz}$. All desorption experiments were performed at room temperature (i.e., $298 \pm 1 \mathrm{~K}$ ). One-mL samples were collected at different time intervals from desorption medium to analyze the kinetic profile of desorption process. Thereafter, the regenerated sorbents were extracted (by filtration for TF; magnetically for MTF), then carefully washed by ultrapure water for reuse in the next run. The desorption efficiency (DE, \%) was calculated according to the following equation:

$D E(\%)=\frac{\text { Amount of desorbed dye }(\mathrm{mmol}) \text { into the eluate }}{\text { Amount of sorbed dye }(\mathrm{mmol})} \times 100$

\section{Results and discussion}

\subsection{Characterization of materials}

\subsubsection{SEM/TEM observations and EDX analysis}

Fig. AM1a (see AMS) shows some SEM micrographs of sorbent particles. The general structure of the sorbents can be described as platelet agglomerated particles (layered material) with irregular objects of different sizes. The morphological aspect is not affected by the incorporation of magnetite: TF and MTF are roughly similar in terms of surface aspect. Fig. AM1b shows the morphology of TF and MTF sorbents after five cycles of sorption and desorption, under ultrasonic treatment. The shape and size of the materials hardly change; the sorbents are remarkably stable under selected experimental conditions. Fig. AM2 shows the EDX spectra of TF and MTF sorbents: nitrogen and sulfur elements are clearly identified in the two materials, while iron is appearing in MTF material; as a confirmation of the incorporation of magnetite into the composite

DLS analysis allows determining the average size of sorbent particles: $0.98 \pm 0.192 \mathrm{~mm}$ for TF and slightly larger at $1.07 \pm 0.215 \mathrm{~mm}$ for MTF (Fig. AM3). In the case of magnetite, the average size of nanoparticles was $6.45 \pm 2.31 \mathrm{~nm}$. Fig. AM4 shows the TEM analysis of magnetite particles. The distribution of size is relatively large; i.e., between 5 and $40 \mathrm{~nm}$. Some of the particles are characterized by a cubic shape while other crystals have a more rounded shape.

\subsubsection{Magnetic properties}

Fig. AM5 shows the vibrating-sample magnetometry analysis of magnetite particles and MTF sorbent. The magnetization saturation is close to $69.1 \mathrm{emu}^{-1}$ for magnetite particles and slightly decreases to $43.8 \mathrm{emu} \mathrm{g}^{-1}$ for the TF-embedded magnetite (MTF). The saturation magnetization of magnetite particles is of the same order of magnitude than the level reported by Zhang et al. [48]; in their case, the immobilization of magnetite particles into poly(urea-formaldehyde) drastically reduced the magnetization saturation (down to $15 \mathrm{~m}^{2} \mathrm{~g}^{-1}$ ). The saturation magnetization of magnetite is frequently reported at values as high as $90-100 \mathrm{emu}^{-1}$. Zawrah et al. [49] found values for magnetite nanoparticles close to $76 \mathrm{emu} \mathrm{g}^{-1}$ and they explain the lower magnetization to the presence of non-magnetic or weakly magnetic interfaces. This clearly supports the decrease of the magnetization after embedment into TF matrix. They also explained that the lower values of magnetization can be explained by (a) incomplete coordination that leads to disordering of magnetic spins, or (b) a weak crystallinity of the magnetite particles. The synthesis of the composite (incorporation of magnetite particles into the TF matrix) may contribute to a similar reduction in crystallinity and magnetization intensity. The magnetization saturation of MTF remains high enough for allowing the magnetic separation of the sorbent from liquid solutions.

\subsubsection{Textural properties}

The textural properties of the studied TF and MTF were characterized using Brunauer, Emmett and Teller (BET) analysis (Table 1). The BET surface area (specific surface area, SSA) reaches $132.6 \mathrm{~m}^{2} \mathrm{~g}^{-1}$ for MTF, this is significantly higher than the specific surface area of TF sorbent (i.e., $111.3 \mathrm{~m}^{2} \mathrm{~g}^{-1}$ ). The incorporation of magnetite microparticles improves the textural properties of the TF-based material. The pore volume $\left(\mathrm{P}_{\mathrm{v}}, \mathrm{cm}^{3} \mathrm{~g}^{-1}\right)$ and the pore size $\left(\mathrm{P}_{\mathrm{R}}, \mathrm{nm}\right)$ are also slightly 
Table 1

Textural properties of TF and MTF sorbents.

\begin{tabular}{lll}
\hline Parameter & \multicolumn{2}{l}{ Sorbent } \\
\cline { 2 - 3 } & TF & MTF \\
\hline Specific surface area $\left(\mathrm{m}^{2} \mathrm{~g}^{-1}\right)$ & 111.3 & 132.6 \\
Pore volume $\left(\mathrm{cm}^{3} \mathrm{~g}^{-1}\right)$ & 0.251 & 0.302 \\
Average pore diameter $(\mathrm{nm})$ & 3.2 & 3.86 \\
Average particle size $(\mathrm{nm})$ & 980 & 1070 \\
\hline
\end{tabular}

increased by the embedment of magnetite nanoparticles within the condensed polymer. Indeed, the average values for pore volume and pore size (calculated by the Barrett-Joyner-Halenda (BJH) desorption method) reach $0.302 \mathrm{~cm}^{3} \mathrm{~g}^{-1}$ and $3.86 \mathrm{~nm}$ for MTF, respectively. This may affect the diffusion properties of the sorbents for the mass transfer of dyes into the polymer network. The embedment of magnetite particles is expected to enhance uptake kinetics. The textural properties of MTF are slightly higher than the values recently reported by Zhao et al. [31] for thiourea-formaldehyde polymer $/ \mathrm{Fe}_{3} \mathrm{O}_{4}$ sorbent (i.e., SSA: $120.6 \mathrm{~m}^{2} \mathrm{~g}^{-1}$; $\mathrm{P}_{\mathrm{V}}: 0.278 \mathrm{~cm}^{3} \mathrm{~g}^{-1}$, and $\mathrm{P}_{\mathrm{R}}: 3.27 \mathrm{~nm}$ ).

\subsubsection{Thermogravimetric analysis}

Fig. AM6 shows the thermogravimetric analysis of TF and MTF. These profiles of thermodegradation are consistent with those reported by previous studies $[33,50]$. The two materials follow the same trend characterized by the following sections:

(a) $25-190{ }^{\circ} \mathrm{C}$ : the weight loss is identical for the two resins that lose about $4 \%$ in weight; this section corresponds to the release of absorbed water (surface moisture and internal water molecules).

(b) $190-380{ }^{\circ} \mathrm{C}$ : TF resin loses about $61 \%$ additional mass $(65 \%$ total weight loss) while MTF only loses 50\% (total weight loss: $54 \%$ ). This step corresponds to the pyrolysis of carbon-skeleton. It is noteworthy that several wavelets can be identified at $229{ }^{\circ} \mathrm{C}, 269{ }^{\circ} \mathrm{C}$ and $319{ }^{\circ} \mathrm{C}$ (the most significant). This probably means that several compounds are successively degraded corresponding to sulfur moieties, amine groups and/or breaking/scission of polymer chains.

(c) $380-650(\mathrm{TF}) / 707(\mathrm{MTF}){ }^{\circ} \mathrm{C}$ : a weak loss of weight is observed (representing a total loss of $76 \%$ for TF and $68 \%$ for MTF).

(d) above $650{ }^{\circ} \mathrm{C}$ (for TF or $707{ }^{\circ} \mathrm{C}$ for MTF) and up to $720{ }^{\circ} \mathrm{C}$ : a new strong loss of mass is observed that represents a total loss of $94.9 \%$ and $71.5 \%$ for TF and MTF, respectively. This phase corresponds to the pyrolysis of the char. It is noteworthy that the temperature range for this ultimate degradation is significantly larger for TF than for MTF (steeper slope characterized by a thin peak on the derivative curve at $710{ }^{\circ} \mathrm{C}$, contrary to the broad band for TF).

These profiles clearly demonstrate the strong attachment of the TF compound to magnetite particles that thermally stabilize the polymer system. In addition, the difference in the residual weights for TF and MTF at complete thermal degradation represents about $23.4 \%$; this is the effective fraction of magnetite in the composite MTF material.

Ahamad and Alsheri [50] analyzed the gas produced during the course of the pyrolysis of thiourea-formaldehyde resin. They identified by FTIR spectrometry, at around $250{ }^{\circ} \mathrm{C}$, the production of sulfur-based gases (i.e.; carbonyl sulfide, carbon disulfide) and ammonia $(\mathrm{N}-\mathrm{H}$ band). At higher temperature (i.e.; $500{ }^{\circ} \mathrm{C}$ ), the pyrolysis is accompanied by the production of hydrogen sulfide, carbon dioxide, ammonia, hydrogen cyanide, carbon disulfide and carbonyl sulfide; the production of nitrogen-based compound being more important as the temperature increases. At $750{ }^{\circ} \mathrm{C}$, their analysis showed the formation of cyanamide and isocyanic acid.

\subsubsection{FTIR and NMR spectroscopies}

The synthesis of the resin is supposed to follow the conventional polycondensation reaction between thiourea and formaldehyde. This is described on Scheme 2; the figure illustrates the different functional groups that could be identified by FTIR spectrometry. Figs. AM7 and AM8 show the FTIR spectra of magnetite, TF and MTF materials at selected wavenumbers $\left(4000-2400 \mathrm{~cm}^{-1}\right.$ and $1800-600 \mathrm{~cm}^{-1}$, respectively). Magnetite is characterized by a large band in the range $3400-3200 \mathrm{~cm}^{-1}$ (assigned to $-\mathrm{OH}$ groups) and some organic impurities (small peaks associated to methyl and methylene groups at $2974 \mathrm{~cm}^{-1}$ and $2922 \mathrm{~cm}^{-1}$ ), a broad band at $1635 \mathrm{~cm}^{-1}$ (attributed to -OH groups). The peaks at $3125 \mathrm{~cm}^{-1}$ (in the broad band), $880 \mathrm{~cm}^{-1}$ and $802 \mathrm{~cm}^{-1}$ are usually characterized as the tracers of $v-\mathrm{OH}, \delta-\mathrm{OH}$, and $\gamma-\mathrm{OH}$ : these two latter bands are diagnostic bands of goethite, hydrated iron oxide compound [51]. The bands at $1090 \mathrm{~cm}^{-1}$ and $1051 \mathrm{~cm}^{-1}$ may be assigned to impurities since they only appear as small peaks in previous studies [52]. The main strong bands for $\mathrm{Fe}-\mathrm{O}$ bonds in magnetite and maghemite are usually identified at $570 \mathrm{~cm}^{-1}$ and $390 \mathrm{~cm}^{-1}$ [51]. The strong absorbance observed at wavenumber below $720 \mathrm{~cm}^{-1}$ does not allow identifying these peaks and wavenumbers on the current FTIR spectrum.

The FTIR spectra of TF and MTF are very similar: most of the peaks represent the superficial organic coating and the bands relative of magnetite particles are not appearing (insignificant changes between TF and MTF). The peaks at $3306 \mathrm{~cm}^{-1}, 1605 \mathrm{~cm}^{-1}$ (and $1135-1132 \mathrm{~cm}^{-1}$ ), $1530-1525 \mathrm{~cm}^{-1}$, and $949 \mathrm{~cm}^{-1}$ are assigned to $>\mathrm{N}-\mathrm{H}$ vibration in secondary amine, $-\mathrm{N}-(\mathrm{C}=\mathrm{S})-,>\mathrm{C}=\mathrm{NH}$<smiles>CCN(CN(CNC(=S)NCO)C(N)=S)C(=S)NCNC(=S)NC</smiles><smiles>CCNC(=S)NC</smiles>

and

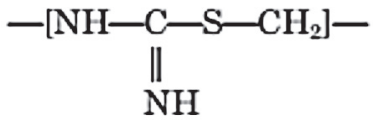

Scheme 2. Tentative structures of TF polymer. 


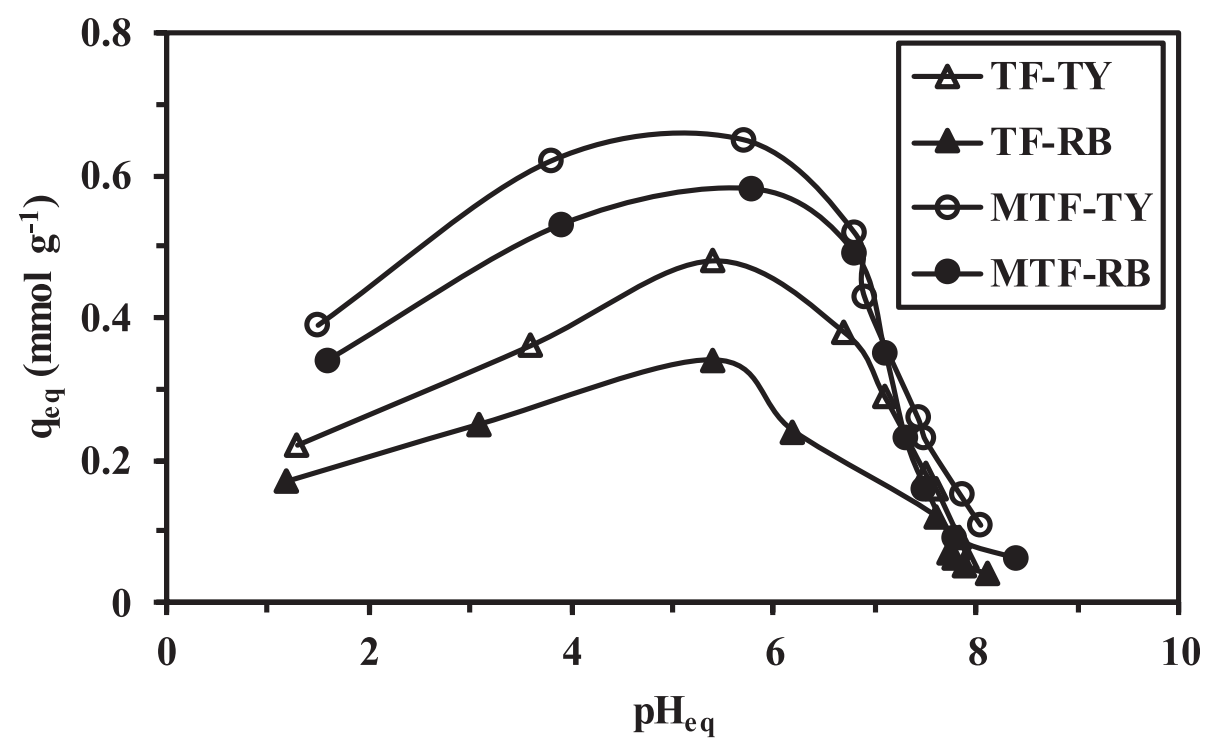

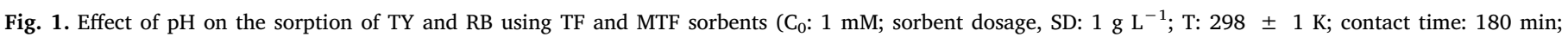
mechanical agitation speed: $210 \mathrm{rpm}$ ).

and > C-S- groups, respectively [34]. The peaks at $1439 \mathrm{~cm}^{-1}$ (and $1320 \mathrm{~cm}^{-1}$ ) are attributed to $\mathrm{C}-\mathrm{N}$ bond [32]. The peak at $1073 \mathrm{~cm}^{-1}$ is attributed to $\mathrm{C}=\mathrm{S}$ stretching vibration and the peak at $1273 \mathrm{~cm}^{-1}$ could be assigned to $\mathrm{C}-\mathrm{H}$ stretching in $>\mathrm{N}-\mathrm{CH}_{2}-\mathrm{N}<$ [37]. The co-existence of these peaks is consistent with the results reported by $\mathrm{Ni}$ et al. [32]; they concluded that two types of chemical structures are simultaneously present, according to Scheme 2.

It is noteworthy that the FTIR spectra of the resins are hardly affected by the binding of TY and RB dyes (Fig. AM9). Similar conclusions were reported by El-Bindary et al. [53].

NMR spectroscopy was used for confirming the chemical structure of TF (Fig. AM10). ${ }^{1} \mathrm{H}$ NMR shows a singlet at $5.05 \mathrm{ppm}$ due to $\mathrm{CH}_{2}$, three $\mathrm{D}_{2} \mathrm{O}$ exchangeable singlet signals at $8.36,8.12$ and $7.36 \mathrm{ppm}$ due to $\mathrm{NH}_{2}, \mathrm{NH}$ and $\mathrm{OH}$ respectively [54]. ${ }^{13} \mathrm{C}$ NMR analysis shows a doublet signal at 184.14 and 184.28 due to $\mathrm{C}=\mathrm{S}$. The DEPT 135 experiment confirmed the presence of $\mathrm{CH}_{2}$. Actually, NMR characterization tends to demonstrate that the most probable structures for TF are represented on Scheme 2 [32,50]. Ahamad [55] proposed a comprehensive mechanism of synthesis for thiourea-formaldehyde resin in acid media, including several steps: (a) protonation of oxygen atom of formaldehyde, (b) formation of monomethylolthiourea, (c) further protonation of the product to form a carbonium ion (growth condensation by water elimination), (d) reaction of the two products with additional thiourea to form methylenedithiourea, and (e) finally polymerization.

\subsection{6. . Surface charge analysis - zetametry}

The sorption properties are strongly affected by the affinity of the sorbent for target solute: chemical affinity (chelating resins, for example) or charge attraction/repulsion (ion-exchange resins). The surface charge is thus a critical parameter that helps in pre-determining the optimum $\mathrm{pH}$ range for solute binding, depending also on the proper acid-base properties of the solute. Fig. AM11 shows the zeta potential of TF and MTF. Surprisingly, the $\mathrm{pH}_{\mathrm{PZC}}$ of the two materials are relatively different, despite the same polymer matrix: the $\mathrm{pH}_{\mathrm{PZC}}$ of $\mathrm{TF}$ decreased from 6.91 to 6.06 when magnetite particles are incorporated in the sorbent. This little shift could be explained by a lower amount of acidbase groups (i.e., S-based and N-based functional groups) on the material because of the presence of the magnetic core (about $23 \%$ of total mass). Anyway, for the two sorbents in acidic solutions the surfaces are positively charged making possible the electrostatic attraction of anionic compounds.

\subsubsection{Stability of materials in aqueous solutions}

The solubility/stability tests of the sorbents immersed into various organic solvents (such as methanol, ethanol, and acetone), acidic solutions (i.e.; $2 \mathrm{~N} \mathrm{HCl}$ ) or alkaline solutions (i.e.; $2 \mathrm{~N} \mathrm{NaOH}$ ) demonstrate that both TF and MTF sorbents are highly stable under these drastic conditions. Actually, the weight loss is not detectable for the different solutions texted, except for $\mathrm{HCl}$ solutions where the weight loss reaches $0.9 \%$. The ultrasound power applied to synthetic resins, such as sulfonated styrene-divinyl benzene resin (Amberlite CR1320) influences the stability of the resin [56] at least at low frequency (i.e., $41 \mathrm{kHz}$ ): increasing the UT power leads to a drastic reduction in the size of resin particles; at higher frequencies $(350 \mathrm{kHz})$, this effect was negligible. This contrasted effect was explained by the lower size of cavitation bubbles at high frequency [57]; lower cavitational collapses (and derived formed microjets) exert weaker mechanical stress on the sorbent [56]. In the present case, the impact of low-frequency UT remains very limited, despite using low-frequency UT (i.e., $35 \mathrm{kHz}$ ).

\subsection{Sorption performances}

\subsection{1. $p H$ effect}

The $\mathrm{pH}$ affects the surface charge of the sorbent; this may be critical especially when the binding mechanism is based on electrostatic attraction: charged surface may attract or repulse ionic solutes. The $\mathrm{pH}$ affects also the protonation/deprotonation of the solute and then its affinity for the sorbent surface. Therefore, optimizing the $\mathrm{pH}$ is the first step in the design of the sorption process. Fig. 1 shows the effect of $\mathrm{pH}$ on the sorption capacities for both TY and RB dyes using TF and MTF sorbents. The four systems follow exactly the same trend: from $\mathrm{pH} 1$ to 5.2-5.8 the sorption progressively increases; between $\mathrm{pH} 5.8$ and 6.8 the sorption capacity stabilizes while above $\mathrm{pH} 6.8$ dye removal decreases and become negligible at $\mathrm{pH}$ close to 8 . It is also important paying attention to $\mathrm{pH}$ variation during the sorption process. Fig. AM12 represents the equilibrium $\mathrm{pH} v s$. initial $\mathrm{pH}$ value. In the range $\mathrm{pH}_{0} 2-6$, the equilibrium $\mathrm{pH}$ increases by up to $2 \mathrm{pH}$ units. The optimum equilibrium $\mathrm{pH}$ 5.2-5.8 corresponds to an initial $\mathrm{pH}$ value close to $\mathrm{pH} 3.2$. This $\mathrm{pH}$ value will be used for further experiments.

At $\mathrm{pH} 3$ (and equilibrium $\mathrm{pH}$ below 5.8), the sorbent is systematically protonated; indeed, Fig. AM11 showed that $\mathrm{pH}_{\mathrm{PZC}}$ values are higher than 6 for both TF and MTF. This means that the sorbents will have electrostatic affinity for anionic forms of the dyes. On the other side, the acid-base properties of the dyes have been evaluated [58]. The 

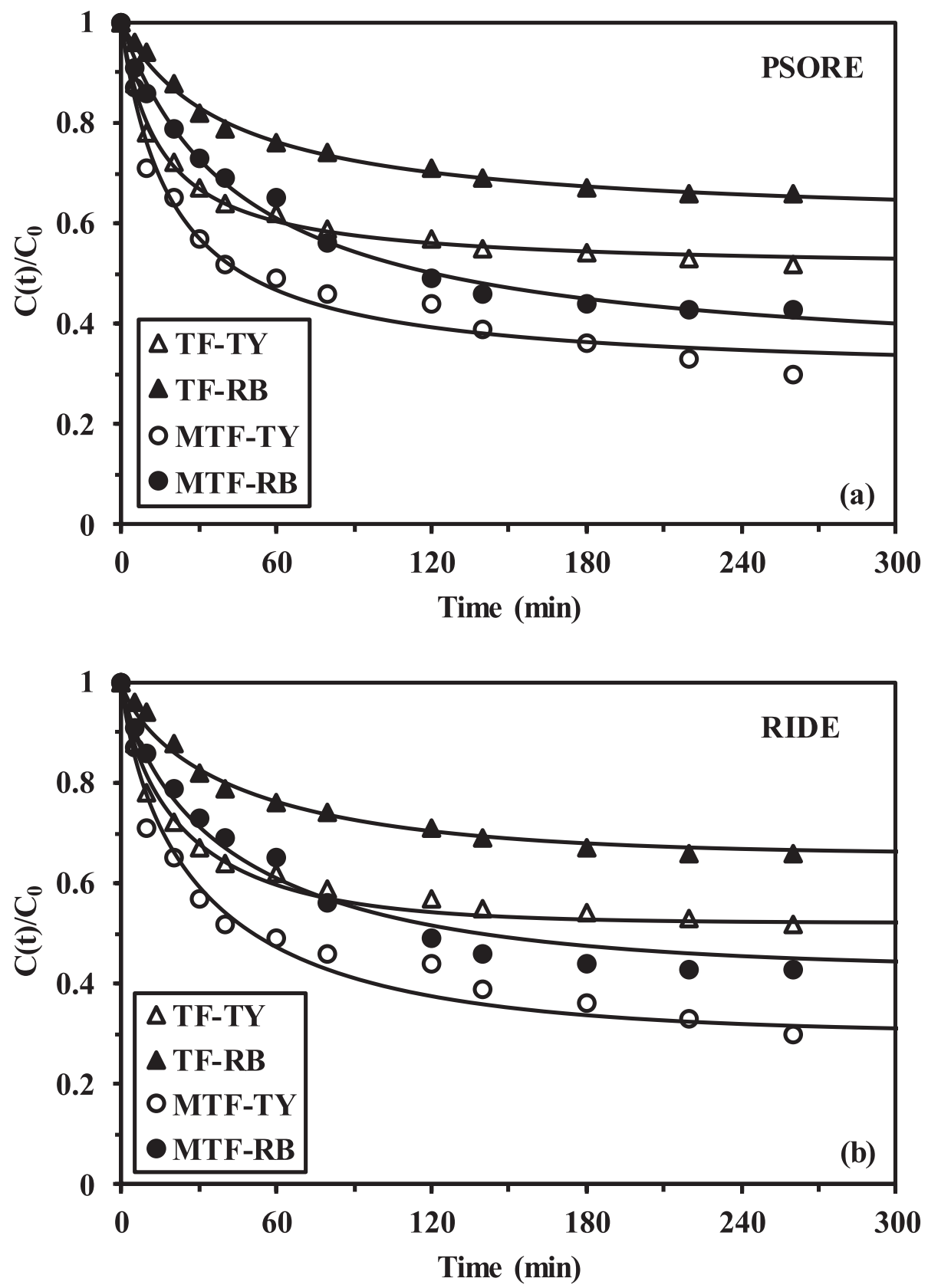

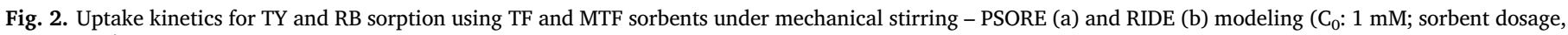
SD: $1 \mathrm{~g} \mathrm{~L}^{-1}$; T: $298 \pm 1 \mathrm{~K}$; mechanical agitation speed: $210 \mathrm{rpm}$ ).

$\mathrm{pK}_{\mathrm{a}}$ values for ionic groups of $\mathrm{RB}$ are respectively 1.89 for the carboxylic/carboxylate group of the dye, while the $\mathrm{pK}_{\mathrm{a}}$ of the hydroxyl groups, hold on phenolic groups, is close to 3.93 [58]. This means that at $\mathrm{pH}$ below 2 the dye is neutral, with limited affinity for protonated reactive groups on TF and MTF sorbents. Between $\mathrm{pH} 2$ and 4, the dye is mono-anionic; this is consistent with the progressive increase of sorption capacity. At $\mathrm{pH}$ above 4, the dye bears two anionic charges, its affinity for protonated amine groups increases and the sorption reaches a maximum because of the good compromise between anionic charges of the dye and the protonated groups on the sorbents. In addition, in this $\mathrm{pH}$ range, there is a limited competition effect of counter anions (brought by the dissociation of the acid used for $\mathrm{pH}$ control at low $\mathrm{pH}$ ). When the $\mathrm{pH}$ increases above 5.8 the protonation of the sorbent decreases and then its affinity for anionic dye species decreases along with sorption capacity. In the case of TY, the reactive groups are the sulfonic/sulfonate moieties. Bao et al [59] reported values around 1 for the
$\mathrm{pK}_{\mathrm{a}}$ of sulfonic groups. The chemical environment and especially the electron-withdrawing of functional groups affect the strength of the acid groups and then their $\mathrm{pK}_{\mathrm{a}}$ but the values remain more acid than carboxylic groups. For $\mathrm{pH}$ above 2 , the deprotonation of sulfonic groups induces a strong affinity for protonated groups on TF and MTF (essentially secondary amine groups and imine groups). The sorption mechanisms for dye are comparable for the two sorbents. The presence of the magnetic core in MTF is not expected to substantially bind the dyes: the coating of magnetite nanoparticles limits the contact with inorganic surface. The $\mathrm{pH}_{\mathrm{PZC}}$ values are of the same order of magnitude (6.91 vs. 6.06 for TF vs. MTF) and much higher than the pH selected for sorption tests. The same reactive groups are present on the two materials and the ion-exchange/electrostatic attraction mechanism is expressing on the same way for the two sorbents at selected $\mathrm{pH}$. The relatively lower density of reactive groups in MTF (due to the presence of magnetite in the composite) is compensated by the little increase in 

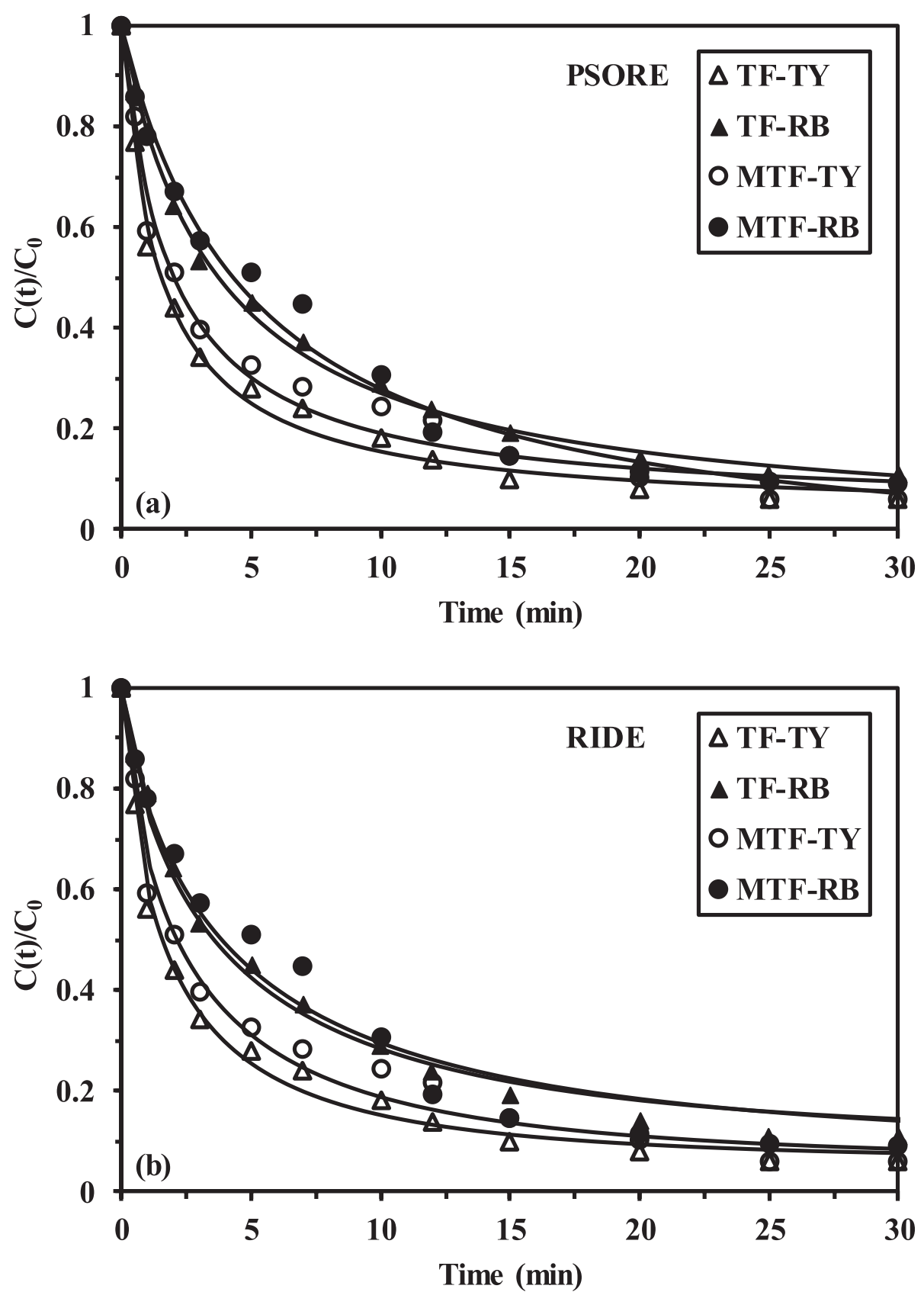

Fig. 3. Uptake kinetics for TY and RB sorption using TF and MTF sorbents under ultrasonic treatment - PSORE (a) and RIDE (b) modeling ( $\mathrm{C}_{0}$ : $1 \mathrm{mM}$; sorbent dosage; $\mathrm{pH}_{0}$ : 3.2; SD: $1 \mathrm{~g} \mathrm{~L}^{-1}$; $\mathrm{T}: 298 \pm 1 \mathrm{~K}$;).

specific surface area and porous volume.

Fig. AM13 shows the plot of the distribution ratio (in log units) vs equilibrium $\mathrm{pH}$. In ion-exchange mechanisms, this plot is frequently used for evaluating the proton exchange involved in the binding mechanism. Each curve representing the system Dye/Sorbent can be separated in two linear sections representing (a) the progressive increase in sorption performance up to the optimum $\mathrm{pH}$ (in the range 5.2-5.8), and (b) a second segment with a higher slope corresponding to the loss in sorption performance, respectively. Table AM2 shows the slope analysis of these different segments. For TF sorbent, the slope is doubled for TY compared with RB and the slope of the second segment is also steeper (doubled) compared with the initial section. In the case of MTF, the difference in the slopes is less marked between TY and RB while the second segment is about 2.6 times the slope of the first segment. In addition, the distribution coefficients of both TY and RB are significantly higher for MTF than for TF. This is first indication of the greater affinity of MTF for the dyes compared to TF; despite the reactive groups are the same and the fact that the actual amount of reactive groups is a little lower than in TF (magnetic core counts for $23 \%$ of total weight). The study of textural properties has shown that the specific surface area is a little higher for MTF over TF. This may contribute to counterbalance the effect of the loss in reactive groups: they are more accessible.

\subsubsection{Uptake kinetics - effect of the mode of agitation}

Figs. 2 and 3 show the kinetic profiles for the sorption of TY and RB, using both TF and MTF sorbents under mechanical agitation and ultrasonic treatment, respectively. The comparison of the curves clearly demonstrates the superior performance of systems submitted to ultrasonic treatment: the equilibrium is reached within $30 \mathrm{~min}$ of contact, while for mechanical agitation the contact time must be extended to about 180-210 min. Similar observations were reported for the sorption 
Table 2

Effect of agitation mode (mechanical agitation (MA) vs. ultrasonic treatment (UT) on the uptake kinetics of TY and RB using TF and MTF sorbents - Model parameters.

\begin{tabular}{|c|c|c|c|c|c|c|}
\hline \multirow[t]{2}{*}{ Sorbent } & \multirow[t]{2}{*}{ Model } & \multirow[t]{2}{*}{ Parameters } & \multicolumn{2}{|l|}{ MA } & \multicolumn{2}{|l|}{ UT } \\
\hline & & & TY & $\mathrm{RB}$ & TY & $\mathrm{RB}$ \\
\hline \multirow[t]{9}{*}{ TF } & Experim. & $\mathrm{q}_{\mathrm{eq}, \exp }\left(\mathrm{mmol} \mathrm{g}^{-1}\right)$ & 0.48 & 0.34 & 0.94 & 0.89 \\
\hline & PFORE & $\mathrm{q}_{\mathrm{eq}, 1}\left(\mathrm{mmol} \mathrm{g}^{-1}\right)$ & 0.444 & 0.331 & 0.875 & 0.854 \\
\hline & & $\mathrm{k}_{1} \times 10^{2}\left(\mathrm{~min}^{-1}\right)$ & 4.93 & 2.25 & 50.7 & 22.4 \\
\hline & & $\mathrm{R}^{2}$ & 0.971 & 0.991 & 0.964 & 0.964 \\
\hline & PSORE & $\mathrm{q}_{\mathrm{eq}, 2}\left(\mathrm{mmol} \mathrm{g}^{-1}\right)$ & 0.494 & 0.401 & 0.971 & 1.01 \\
\hline & & $\begin{array}{l}\mathrm{k}_{2} \times 10^{2} \\
\left(\mathrm{~g} \mathrm{mmol}^{-1} \mathrm{~min}^{-1}\right)\end{array}$ & 13.6 & 5.99 & 69.8 & 26.0 \\
\hline & & $\mathrm{R}^{2}$ & 0.996 & 0.995 & 0.993 & 0.997 \\
\hline & RIDE & $\mathrm{D}_{\mathrm{e}} \times 10^{10}\left(\mathrm{~m}^{2} \min ^{-1}\right)$ & 12.7 & 7.87 & 22.1 & 18.5 \\
\hline & & $\mathrm{R}^{2}$ & 0.990 & 0.992 & 0.992 & 0.994 \\
\hline \multirow[t]{9}{*}{ MTF } & Experim. & $\mathrm{q}_{\mathrm{eq}, \exp }\left(\mathrm{mmol} \mathrm{g}^{-1}\right)$ & 0.70 & 0.57 & 0.942 & 0.909 \\
\hline & PFORE & $\mathrm{q}_{\mathrm{eq}, 1}\left(\mathrm{mmol} \mathrm{g}^{-1}\right)$ & 0.621 & 0.569 & 0.851 & 0.901 \\
\hline & & $\mathrm{k}_{1} \times 10^{2}\left(\min ^{-1}\right)$ & 4.02 & 1.98 & 41.4 & 17.5 \\
\hline & & $\mathrm{R}^{2}$ & 0.954 & 0.991 & 0.949 & 0.981 \\
\hline & PSORE & $\mathrm{q}_{\mathrm{eq}, 2}\left(\mathrm{mmol} \mathrm{g}^{-1}\right)$ & 0.704 & 0.693 & 0.960 & 1.085 \\
\hline & & $\begin{array}{l}\mathrm{k}_{2} \times 10^{2} \\
\left(\mathrm{~g} \mathrm{mmol}^{-1} \mathrm{~min}^{-1}\right)\end{array}$ & 7.30 & 3.08 & 55.0 & 18.2 \\
\hline & & $\mathrm{R}^{2}$ & 0.984 & 0.993 & 0.984 & 0.986 \\
\hline & RIDE & $\mathrm{D}_{\mathrm{e}} \times 10^{10}\left(\mathrm{~m}^{2} \mathrm{~min}^{-1}\right)$ & 6.78 & 6.68 & 18.5 & 16.5 \\
\hline & & $\mathrm{R}^{2}$ & 0.981 & 0.993 & 0.985 & 0.976 \\
\hline
\end{tabular}

of malachite green by biosorbents; both the sorption capacity at equilibrium and the mass transfer were enhanced by applying ultrasonic treatment [40]. The cavitation bubbles created during the ultrasonic treatment produce high-speed micro-jets and high-pressure shocks that contribute to improve mass transfer properties [28,41,42]. This is also attributed to thermal effects (creation of local "hot spots" due to cavitation shocks) that either alter the porous structure or change the activation energy of the sorption process.

The kinetic profiles are fitted using the PFORE, PSORE and RIDE equations (Table AM1). The solid lines on Figs. 2 and 3 represent the mathematical fits for PSORE and RIDE while the PFORE modeling is represented on Fig. AM14 with parameters summarized in Table 2. The comparisons of the determination coefficients $\left(\mathrm{R}^{2}\right)$ and the fitting trends (on figures) clearly demonstrate that the PSORE is more appropriate than the PFORE for modeling kinetic profiles. It is noteworthy that the calculated value of the sorption capacity at equilibrium overestimates the corresponding experimental value but remains in the same order of magnitude (except for RB under mechanical agitation for which the overestimation exceeds $20 \%$ ). It is generally accepted that the PSORE corresponds to systems controlled by surface chemisorption [60]. The comparison of sorption capacities at equilibrium confirms two trends: (a) higher sorption capacities for MTF compared to TF and (b) enhancement of sorption capacities with ultrasonic treatment. The TY dye shows (indifferently for PFORE and PSORE) apparent rate coefficients two times greater than for RB dye; the same trends are observed for mechanical and ultrasonic agitations. This can be due to a higher reactivity of TY compared with RB, because this dye bears two sulfonic reactive groups, which are easily accessible at the end of the linear molecule, while RB may suffer from hindrance effects for accessing to the single carboxylic reactive group in the compact molecule of the dye. Contrary to the trend followed by sorption capacities at equilibrium the apparent rate coefficients are little lower for MTF sorbent than for TF (indifferently for mechanical and ultrasonic agitation modes). On the other hand, the ultrasonic treatment considerably increases the values of the apparent rate coefficients by a factor between 7 and 10, depending on the system (dye/sorbent); the same trend is observed for both $\mathrm{k}_{1}$ (PFORE) and $\mathrm{k}_{2}$ (PSORE).

Although the RIDE is a little less accurate in the description of kinetic profiles than PSORE the fitting is relatively good (as shown on
Figs. 2 and 3) and the resistance to intraparticle diffusion is playing a role in the control of uptake kinetics. The comparison of the effective diffusivity coefficients $\left(D_{e}, \mathrm{~m}^{2} \mathrm{~min}^{-1}\right)$ shows that: (a) the diffusivity is a little lower for RB than for TY, (b) the diffusion properties are unexpectedly weaker for MTF compared to TF (despite little higher porosity), (c) the ultrasonic treatment strongly increases diffusion.

Fig. AM15 compares the sorption capacities at 15 min of contact for experiments performed under mechanical agitation and under the combined mechanical and ultrasonic agitation at different speeds of agitation. As expected, the ultrasonic treatment improves the sorption capacities. The sorption capacity linearly increases with agitation speed for the different systems; although at high agitation speed (around $200 \mathrm{rpm}$ ), the increase in $\mathrm{q}_{15 \mathrm{~min}}$ tends to stabilize. This means that the resistance to film diffusion plays a non-negligible role in the control of global mass transfer; however, at $200 \mathrm{rpm}$ this effect becomes progressively negligible.

In their discussion of the effects of ultrasound treatment on sorption properties, Breitbach and Bathen [56] demonstrated the increase of the pore diffusion coefficient with both the power and the frequency of the ultrasound system. Mass transfer in the pores is clearly increased because of the enhancement of turbulence and convective transport inside the pores due to microjets formation (growing and collapse of cavitation bubbles with sound cycles). The present results are consistent with this mechanism. Substantial increases (up to 8-10 times) in diffusion coefficients were reported for the binding of organic pollutants onto activated carbon while applying ultrasonication [57]. The beneficial effect of ultrasonic treatment was also attributed to the decrease of the boundary layer surrounding sorbent particles (associated with resistance to film diffusion mechanism) [57]. Sarkar and Sarkar [61] also reported similar increases in kinetic constants for the sorption of $\mathrm{Cr}(\mathrm{VI})$ onto iron(III)-cellulose nanocomposite submitted to ultrasound assisted sorption (compared with conventional agitation system).

\subsubsection{Sorption isotherms}

The sorption isotherms represent the equilibrium distribution of the solute between the liquid and solid phases for increasing concentrations, at fixed temperatures and pH. Figs. 4 and 5 show the experimental profiles for TY and RB dyes using both TF and MTF under mechanical or ultrasonic agitation at room temperature (i.e., $298 \pm 1 \mathrm{~K}$ ) and for an initial $\mathrm{pH}$ of 3.2 (equilibrium $\mathrm{pH}$ values in the range:5.3-5.7). Under mechanical agitation, the sorption of both TY and RB is higher with MTF compared with TF (consistently with kinetic results). It is noteworthy that for TF sorbent the initial slope of the curve (which is representative of the affinity of the sorbent for the dye) is overlapped for the two dyes, contrary to MTF sorbent; the initial slope is steeper for TY than for RB. A hypothesis has been reported above in relation with the number of anionic reactive groups hold on the dyes. Under ultrasonic treatment, the profiles are completely different. The two dyes have exactly the same behavior: the curves are superimposed for both TF and MTF: they have the same affinity (initial slope) and the same saturation levels. The ultrasonic treatment tends to minimize the impact of the chemical structure or molecular size of the dyes. However, the affinity (initial slope) remains higher for MTF than for $\mathrm{TF}$.

The ultrasound treatment is supposed to favor the desorption mechanism against sorption process [56]. Therefore, if the shape of the isotherm is supposed to be maintained, a shift towards lower surface concentrations may occur. Actually, this is not the case for the present system. Indeed, higher saturation levels are reached with ultrasonic treatment; in addition, saturation occurs at much lower residual dye concentrations. This probably means that the UT improves the accessibility to internal sites and that this effect predominates against desorption enhancement.

The sorption isotherms were modeled using the equations of Langmuir, Freundlich, Sips and Temkin (Table AM2). Table 3 reports the parameters of the models and their relevant determination 

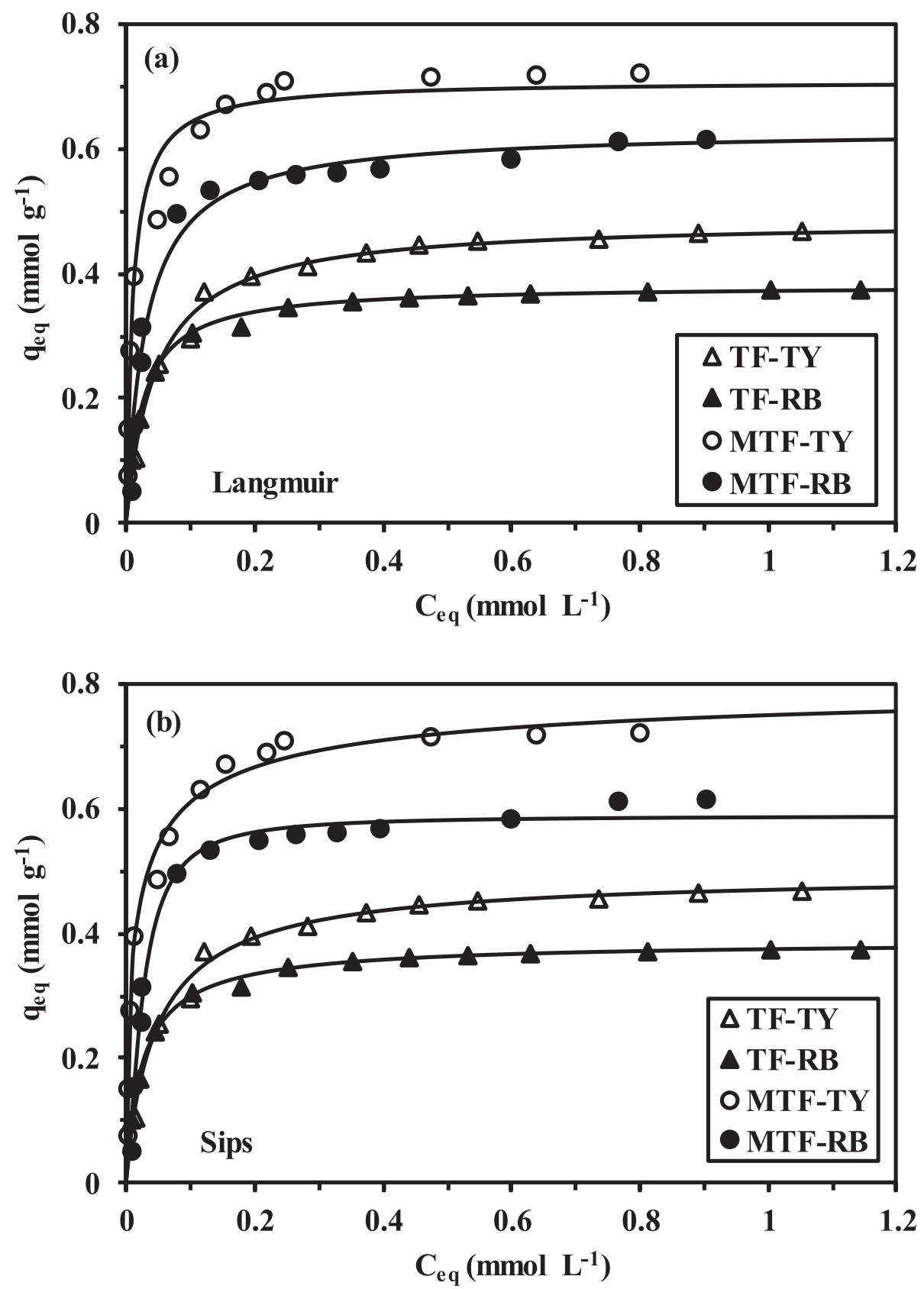

Fig. 4. Sorption isotherms for TY and RB sorption using TF and MTF sorbents under mechanical stirring- Langmuir (a) and Sips (b) modeling $\left(\mathrm{C}_{0}\right.$ : $0.0 .06-1 \mathrm{mM}$; $\mathrm{pH}$ : 3.0; sorbent dosage, SD: $1 \mathrm{~g} \mathrm{~L}^{-1}$; T: $298 \pm 1 \mathrm{~K}$; contact time: $180 \mathrm{~min}$; mechanical agitation speed: $210 \mathrm{rpm}$ ).

coefficients. Figs. 4 and 5 show the fits of experimental profiles using the Langmuir and the Sips equations while Figs. AM16 and AM17 present the fits with the Freundlich and Temkin equations. The best fits were obtained using the Langmuir and the Sips equations: the incorporation of a third adjustable parameter in the Sips equation logically increases the quality of the mathematical fit, at the expense of a loss in the physical significance of the sorption capacity. The Langmuir equation tends to overestimate the sorption capacity at saturation of the monolayer. The maximum sorption capacities are higher for TY than for $\mathrm{RB}$, The sorption capacities for MTF are higher than for MF under mechanical agitation, while for ultrasonic agitation the differences tend to be less marked. The affinity coefficients (b parameter of the Langmuir equation and initial slope) are strongly increased under ultrasonic agitation, by 5 to 8 times (and up to 25 times for RB/MTF system). For TF sorbent, the affinity coefficient is higher for RB than for TY, while a reciprocal trend is observed for MTF under mechanical agitation; the ultrasonic treatment tends to minimize the differences for MTF (contrary to $\mathrm{TF}$ ).

The Temkin equation offers the possibility to approach energetic aspects: the sorption heat is supposed to increase linearly with sorption capacity [62]. The energetic coefficient $B_{T}\left(\mathrm{~J} \mathrm{~mol}^{-1}\right)$ significantly decreases with ultrasonic treatment compared to mechanical agitation meaning that less energy is required for solute binding. In addition, the coefficient $\mathrm{B}_{\mathrm{T}}$ also decreases when using MTF instead of TF: this confirms the higher affinity of the magnetic sorbent compared to raw resin (despite a lower density of reactive groups). On the other hand, the other constant of the Temkin model (i.e., $\mathrm{A}_{\mathrm{T}}, \mathrm{L} \mathrm{mmol}^{-1}$ ) tends to: (a) increase with ultrasonic treatment, (b) be higher for MTF (compared to $\mathrm{TF}$ ), and (c) be higher for RB (compared to TF); the exception being RB sorption on TF sorbent under ultrasonic agitation.

In addition to cavitation and thermal effects, another action of the ultrasonic treatment may consist of increasing the kinetic energy of dye 

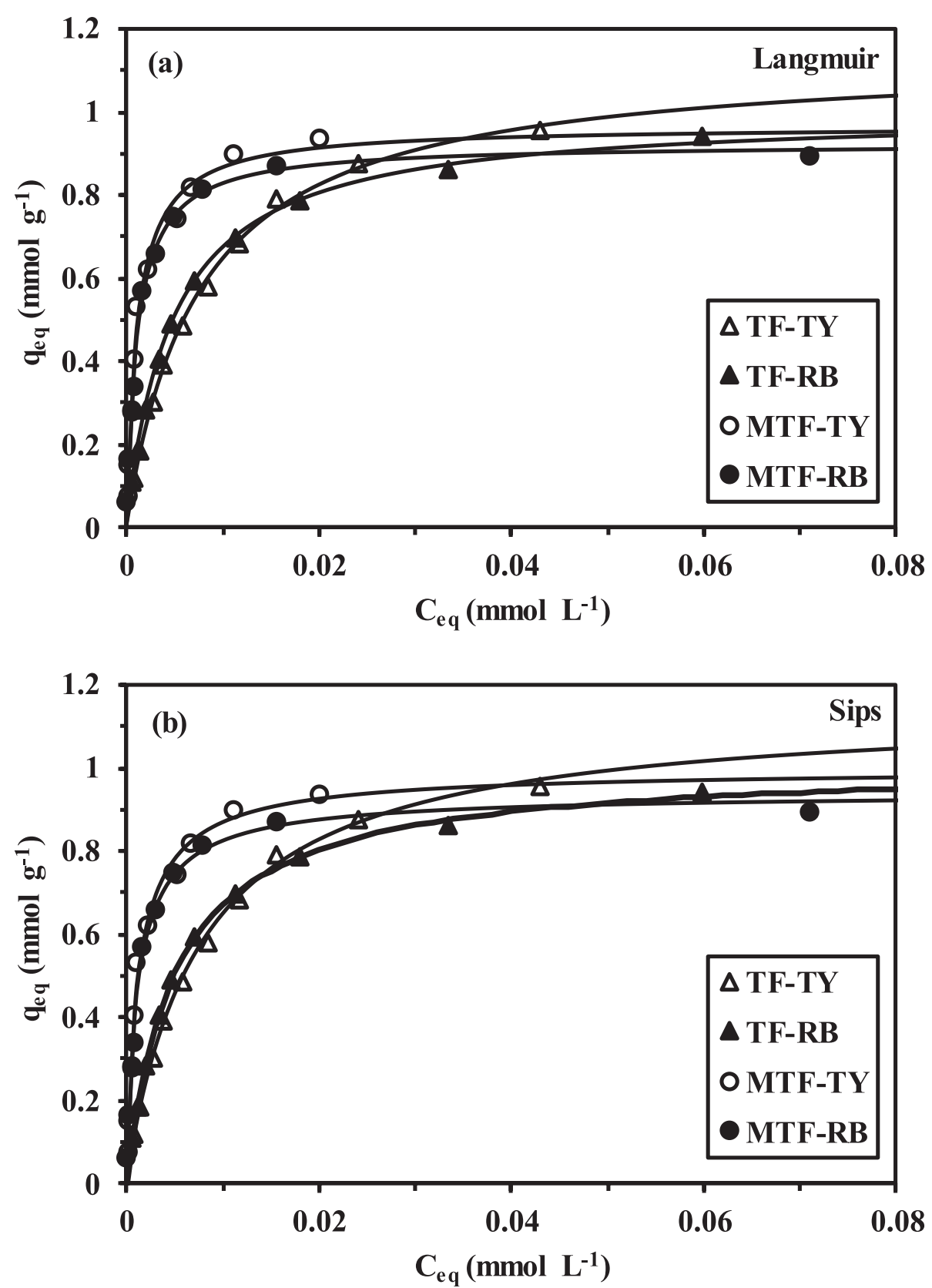

Fig. 5. Sorption isotherms for TY and RB sorption using TF and MTF sorbents under ultrasonic treatment - Langmuir (a) and Sips (b) modeling $\left(\mathrm{C}_{0}\right.$ : $0.0 .06-1$ mM; $\mathrm{pH}_{0}$ : 3.0; SD: $1 \mathrm{~g} \mathrm{~L}^{-1}$; $\mathrm{T}: 298 \pm 1 \mathrm{~K}$; contact time: $30 \mathrm{~min}$.

molecules, which, in turn, may overcome the steric hindrance associated with the large size of dye molecules.

\subsubsection{Thermodynamic parameters}

The sorption capacities for TY and RB on TF and MTF were compared at different temperatures (in the range 298-328 K) for fixed experimental conditions (i.e., $\mathrm{C}_{0}: 1 \mathrm{mmol} \mathrm{L}^{-1}$; $\mathrm{pH} \mathrm{3,} \mathrm{SD:} 1 \mathrm{~g} \mathrm{~L}^{-1}$ ). The sorption capacities progressively decrease with increasing the temperature: the sorption process is exothermic.

The Van't Hoff equation (Eq. (3)) was used for calculating the thermodynamic parameters; i.e., enthalpy change: $\Delta \mathrm{H}^{\circ}\left(\mathrm{kJ} \mathrm{mol}^{-1}\right)$, free energy change: $\Delta \mathrm{G}^{\circ}\left(\mathrm{kJ} \mathrm{mol}{ }^{-1}\right)$ and entropy change: $\Delta \mathrm{S}^{\circ}$ (kJ $\mathrm{mol}^{-1} \mathrm{~K}^{-1}$ ).

$\ln D=\frac{-\Delta H^{\circ}}{R T}+\frac{-\Delta S^{\circ}}{T}$
$\Delta G^{\circ}=\Delta H^{\circ}-T \Delta S^{\circ}$

Fig. AM18 shows the plot of $\ln \mathrm{D}$ (i.e., $\mathrm{q}_{\mathrm{eq}} / \mathrm{C}_{\mathrm{eq}}$ ) vs. the reciprocal of absolute temperature (i.e., 1/T). Slope and ordinate intercept were used for the determination of $\Delta \mathrm{H}^{\circ}, \Delta \mathrm{G}^{\circ}$ and $\Delta \mathrm{S}^{\circ}$. In terms of distribution ratios, under mechanical agitation the systems can be ranked according the series: MTF-TY > MTF-RB > TF-TY > TF-RB. Under ultrasonic treatment, a complete change is observed: all the systems behave very closely, except for MTF-RB that is characterized by significantly lower distribution ratios. However, the ultrasonic treatment shifts the distribution ratios by about two orders of magnitude compared with mechanical agitation. The beneficial effect of ultrasonic treatment on metal sorption is clearly confirmed. Table 4 summarizes thermodynamic parameters. As expected, the enthalpy change is negative (from -6.43 to $-14.6 \mathrm{~kJ} \mathrm{~mol}^{-1}$ ): the sorption process is exothermic. The absolute value of the enthalpy change is higher for MTF than for MF for mechanical agitation (MA), while a reciprocal trend is observed 
Table 3

Effect of agitation mode (mechanical agitation (MA) vs. ultrasonic treatment (UT) on the sorption isotherms of TY and RB using TF and MTF sorbents Model parameters.

\begin{tabular}{|c|c|c|c|c|c|c|}
\hline \multirow[t]{2}{*}{ Sorbent } & \multirow[t]{2}{*}{ Model } & \multirow[t]{2}{*}{ Parameters } & \multicolumn{2}{|l|}{ MA } & \multicolumn{2}{|l|}{ UT } \\
\hline & & & TY & $\mathrm{RB}$ & TY & $\mathrm{RB}$ \\
\hline \multirow[t]{11}{*}{ TF } & Experim. & $\mathrm{q}_{\mathrm{eq}, \exp }\left(\mathrm{mmol} \mathrm{g}^{-1}\right)$ & 0.453 & 0.369 & 0.957 & 0.939 \\
\hline & \multirow[t]{3}{*}{ Langmuir } & $\mathrm{q}_{\mathrm{eq}, \mathrm{L}}\left(\mathrm{mmol} \mathrm{g}^{-1}\right)$ & 0.487 & 0.380 & 1.14 & 1.00 \\
\hline & & $\mathrm{b}_{\mathrm{L}}\left(\mathrm{L} \mathrm{mmol}^{-1}\right)$ & 21.5 & 39.1 & 131.5 & 206.5 \\
\hline & & $\mathrm{R}^{2}$ & 0.987 & 0.995 & 0.998 & 0.999 \\
\hline & \multirow[t]{4}{*}{ Sips } & $\mathrm{q}_{\mathrm{eq}, \mathrm{s}}\left(\mathrm{mmol} \mathrm{g}^{-1}\right)$ & 0.497 & 0.388 & 1.15 & 1.02 \\
\hline & & $\mathrm{b}_{\mathrm{s}}\left(\mathrm{L} \mathrm{mmol}{ }^{-1}\right)$ & 16.6 & 26.1 & 122.9 & 165.1 \\
\hline & & $\mathrm{n}_{\mathrm{S}}$ & 1.08 & 1.11 & 1.01 & 1.04 \\
\hline & & $\mathrm{R}^{2}$ & 0.987 & 0.997 & 0.998 & 1.0 \\
\hline & \multirow[t]{3}{*}{ Temkin } & $\mathrm{A}_{\mathrm{T}}\left(\mathrm{L} \mathrm{mmol}{ }^{-1}\right)$ & 401.8 & 1368 & 3847 & 321.8 \\
\hline & & $\mathrm{B}_{\mathrm{T}}\left(\mathrm{J} \mathrm{mol}^{-1}\right)$ & 29,741 & 45,121 & 24,342 & 19,254 \\
\hline & & $\mathrm{R}^{2}$ & 0.979 & 0.969 & 0.989 & 0.934 \\
\hline \multirow[t]{11}{*}{ MTF } & Experim. & $q_{\text {eq,exp }}\left(\mathrm{mmol} \mathrm{g}^{-1}\right)$ & 0.708 & 0.568 & 0.935 & 0.893 \\
\hline & \multirow[t]{3}{*}{ Langmuir } & $\mathrm{q}_{\mathrm{eq}, \mathrm{L}}\left(\mathrm{mmol} \mathrm{\textrm {g } ^ { - 1 } )}\right.$ & 0.708 & 0.631 & 0.968 & 0.925 \\
\hline & & $\mathrm{b}_{\mathrm{L}}\left(\mathrm{L} \mathrm{mmol}^{-1}\right)$ & 96.0 & 32.2 & 869.1 & 866.1 \\
\hline & & $\mathrm{R}^{2}$ & 0.969 & 0.976 & 0.966 & 0.987 \\
\hline & \multirow[t]{4}{*}{ Sips } & $\mathrm{q}_{\mathrm{eq}, \mathrm{s}}\left(\mathrm{mmol} \mathrm{g}^{-1}\right)$ & 0.814 & 0.589 & 1.00 & 0.942 \\
\hline & & $\mathrm{b}_{\mathrm{S}}\left(\mathrm{L} \mathrm{mmol}{ }^{-1}\right)$ & 11.7 & 228.6 & 458.1 & 484.0 \\
\hline & & $\mathrm{n}_{\mathrm{S}}$ & 1.68 & 0.68 & 1.09 & 1.09 \\
\hline & & $\mathrm{R}^{2}$ & 0.989 & 0.987 & 0.966 & 0.985 \\
\hline & \multirow[t]{3}{*}{ Temkin } & $\mathrm{A}_{\mathrm{T}}\left(\mathrm{L} \mathrm{mmol}{ }^{-1}\right)$ & 5049 & 465 & 15,800 & 65,776 \\
\hline & & $\mathrm{B}_{\mathrm{T}}\left(\mathrm{J} \mathrm{mol}^{-1}\right)$ & 26,417 & 22,283 & 14,715 & 21,313 \\
\hline & & $\mathrm{R}^{2}$ & 0.990 & 0.945 & 0.944 & 0.912 \\
\hline
\end{tabular}

in the case of ultrasonic treatment (UT). However, the variations are not marked enough to make a definitive differentiation between the two systems. In the case of RB dye, the ultrasonic treatment slightly decreased the absolute value of the enthalpy change while for TY dye the evolution is not homogeneous between TF (increase) and MTF (decrease). It is this difficult assigning the ultrasonic treatment a clear effect on the enthalpy change for the different sorbent/dye systems. The entropy change is also negative (ranging between -25 and $-38 \mathrm{~J} \mathrm{~mol}^{-1} \mathrm{~K}^{-1}$ ) for mechanical agitation; this means that the sorption contributes to decrease the randomness of the system due to the arrangement of dyes molecules at the surface of the sorbents. In the case of ultrasonic treatment, the entropy changes are negative (varying between -2.89 and $-16.3 \mathrm{~J} \mathrm{~mol}^{-1} \mathrm{~K}^{-1}$ ), except for MTF-TY, where the entropy change is slightly positive. The absolute values of the entropic term $\left(\left|\mathrm{T} \Delta \mathrm{S}^{\circ}\right|\right)$ being strictly inferior to the enthalpy $\left(\left|\Delta \mathrm{H}^{\circ}\right|\right)$, the system can be considered driven by enthalpy rather than by entropy in the case of ultrasonic treatment while a reciprocal trend is observed for mechanical agitation (entropic control). The free energy changes are positive for most of the systems (except for mechanical agitation with MTF and TY dye and for ultrasonic treatment with TF and RB dye): the sorption is spontaneous in most cases (contrary to the two cited exception). No reason was found to explain these unexpected differences for these two specific cases.
An additional experiment was performed comparing the sorption capacities reached after $30 \mathrm{~min}$ of contact for the different systems with mechanical agitation, ultrasonic treatment at $35 \mathrm{kHz}$ and $40 \mathrm{kHz}$. This is summarized in Table AM4. Applying the ultrasonic treatment significantly improves dye sorption for the different systems; in addition, increasing ultrasonic frequency slightly increases the sorption capacities. The difference in UT power is not marked enough for exhibiting substantial differences as reported by Breitbach and Bathen [56]. While comparing conventional and ultrasound-assisted sorption of $\mathrm{Cr}(\mathrm{VI})$ onto composite iron(III)-cellulose, it was also demonstrated that the free Gibbs energy $\left(-\Delta G^{\circ}\right)$ increased with UT [61]. On the opposite hand, Sarkar and Sarkar found positive values of change in entropy that increase with applying UT. Here, the negative values of entropy change are progressively levelled of using UT (up to reaching positive value for the MTF-TY system).

\subsubsection{Effect of co-existing/competitor anions}

The sorption performance can be strongly affected by the composition of the solution (presence of ligands or competitor ions), especially for systems involving ion-exchange processes. In order to evaluate the selectivity or stability of sorption performances, the sorption capacity for TY on TF sorbent was tested in the presence of increasing concentrations of competitor anions (Fig. 6). As expected, the sorption capacity decreases with the concentration of competitor anions. However, even in the presence of a large excess of anions (up to $1.8 \mathrm{~mol} \mathrm{~L}^{-1}$ $v s$. a dye concentration of $1 \mathrm{mmol} \mathrm{L}^{-1}$ ) sorption capacity is maintained at high levels: $0.21-0.39 \mathrm{mmol} \mathrm{TY} \mathrm{g}^{-1}$ vs. $0.48 \mathrm{mmol} \mathrm{TY} \mathrm{g}^{-1}$ in the absence of competitor anions. The competitor effects follows the series: nitrate $<$ sulfate $<$ chloride $<$ phosphate. This is illustrated by Fig. AM19; the plots of the distribution coefficients as a function of the initial concentration of competitor ions ( $\log _{10}$ units) show linear trends with increasing slopes from nitrate to phosphate (from -0.178 to -0.569 ). At $\mathrm{pH}$ close to 5 (equilibrium $\mathrm{pH}$ value), sulfate and phosphate ions are essentially present as $\mathrm{HSO}_{4}{ }^{-}$and phosphate as $\mathrm{HPO}_{4}{ }^{2-}$ species. The ranking of competitor ions is not directly correlated to the ionic charge of the competitor anions. In the case of the sorption of sulfonic-based dyes on anion exchangers, Karcher et al. [63] also reported that sulfate anions had no significant effect on sorption performance while chloride ions slightly influenced sorption capacity but much less than phosphate anions. They commented that the more pronounced effect of phosphate could be due to higher negative charge. It is important to take into account that strong ionic strengths may affect the conformation of organic compounds in solutions (including organic dyes) due to screening effects and coiling mechanisms [64]. Mahmoodi et al. [65] referred to the small size of inorganic anions for explaining the competition effect on sulfonic-bearing anionic dyes on biosorbents. The hydrated volumes of competitor anions, $\mathrm{V}_{\mathrm{Hydr}}$ $\left(\mathrm{cm}^{3} \mathrm{~mol}^{-1}\right)$, follow the series: nitrate $(23.3)<$ chloride $(34.5) \approx$ hydrogen phosphate (34.6) < hydrogen sulfate (41.2) [66], which is not consistent with the competitor ranking scale observed above. The scale of ionic radii, $\mathrm{R}_{\mathrm{i}}(\mathrm{pm})$ also fails to correlate with this competitor order:

Table 4

Thermodynamic parameters for the sorption of TY and RB on TF and MTF sorbent under mechanical agitation (MA) or ultrasonic treatment (UT).

\begin{tabular}{|c|c|c|c|c|c|c|c|c|c|c|c|c|c|}
\hline \multirow[t]{2}{*}{ Agitation mode } & \multirow[t]{2}{*}{ Sorbent } & \multirow[t]{2}{*}{ Dye } & \multirow[t]{2}{*}{$\Delta \mathrm{H}^{0}\left(\mathrm{~kJ} \mathrm{~mol}^{-1}\right)$} & \multirow[t]{2}{*}{$\Delta S^{0}\left(\mathrm{~J} \mathrm{~mol}^{-1} \mathrm{~K}^{-1}\right)$} & \multirow[t]{2}{*}{$\mathrm{R}^{2}$} & \multicolumn{4}{|c|}{$\mathrm{T} \Delta \mathrm{S}^{0}\left(\mathrm{~kJ} \mathrm{~mol}^{-1}\right)$} & \multicolumn{4}{|c|}{$\Delta \mathrm{G}^{0}\left(\mathrm{~kJ} \mathrm{~mol}^{-1}\right)$} \\
\hline & & & & & & $298 \mathrm{~K}$ & $308 \mathrm{~K}$ & $318 \mathrm{~K}$ & $328 \mathrm{~K}$ & $298 \mathrm{~K}$ & $308 \mathrm{~K}$ & $318 \mathrm{~K}$ & $328 \mathrm{~K}$ \\
\hline \multirow[t]{4}{*}{ MA } & \multirow[t]{2}{*}{$\mathrm{TF}$} & TY & -9.25 & -35.0 & 0.949 & -10.35 & -10.65 & -10.99 & -11.34 & 1.05 & 1.39 & 1.75 & 2.09 \\
\hline & & $\mathrm{RB}$ & -8.93 & -38.0 & 0.997 & -11.38 & -11.76 & -12.14 & -12.52 & 2.45 & 2.83 & 3.21 & 3.59 \\
\hline & \multirow[t]{2}{*}{ MTF } & TY & -14.6 & -25.0 & 0.992 & -7.49 & -7.74 & -8.00 & -8.25 & -7.12 & -6.86 & -6.61 & -6.36 \\
\hline & & $\mathrm{RB}$ & -9.30 & -34.0 & 0.984 & -10.06 & -10.40 & -10.73 & -11.07 & 0.76 & 1.09 & 1.43 & 1.77 \\
\hline \multirow[t]{4}{*}{ UT } & \multirow[t]{2}{*}{$\mathrm{TF}$} & TY & -12.0 & -16.3 & 0.926 & -4.86 & -5.02 & -5.19 & -5.35 & -7.16 & -6.99 & -6.83 & -6.67 \\
\hline & & $\mathrm{RB}$ & -7.69 & -2.89 & 0.973 & -0.86 & -0.89 & -0.92 & -0.95 & -6.83 & -6.80 & -6.77 & -6.75 \\
\hline & \multirow[t]{2}{*}{ MTF } & TY & -6.43 & 1.26 & 0.950 & 0.38 & 0.39 & 0.40 & 0.41 & -6.80 & -6.82 & -6.83 & -6.84 \\
\hline & & $\mathrm{RB}$ & -7.00 & -6.07 & 0.948 & -1.81 & -1.87 & -1.93 & -1.99 & -5.19 & -5.12 & -5.06 & -5.00 \\
\hline
\end{tabular}




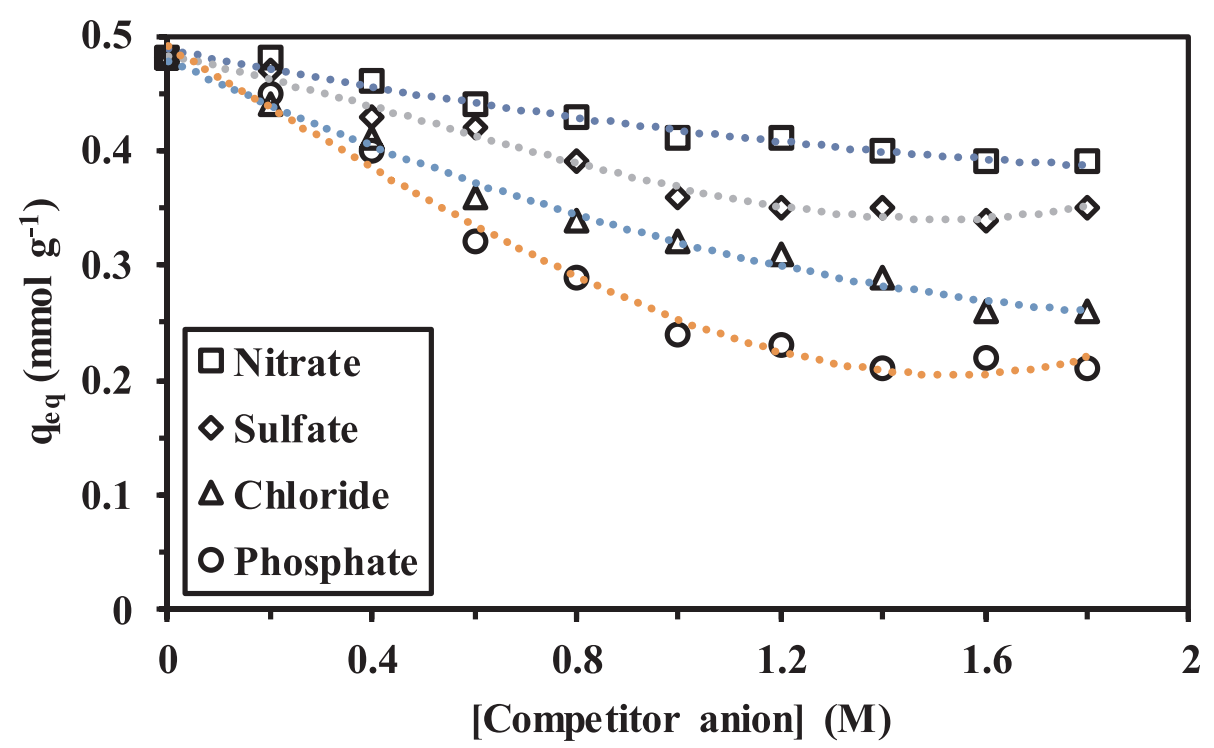

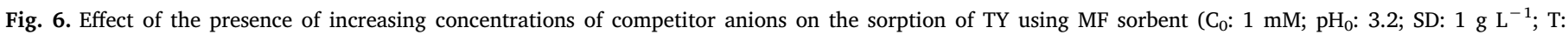
$298 \pm 1 \mathrm{~K}$; agitation time: $180 \mathrm{~min}$; mechanical agitation speed: $210 \mathrm{rpm}$ ).
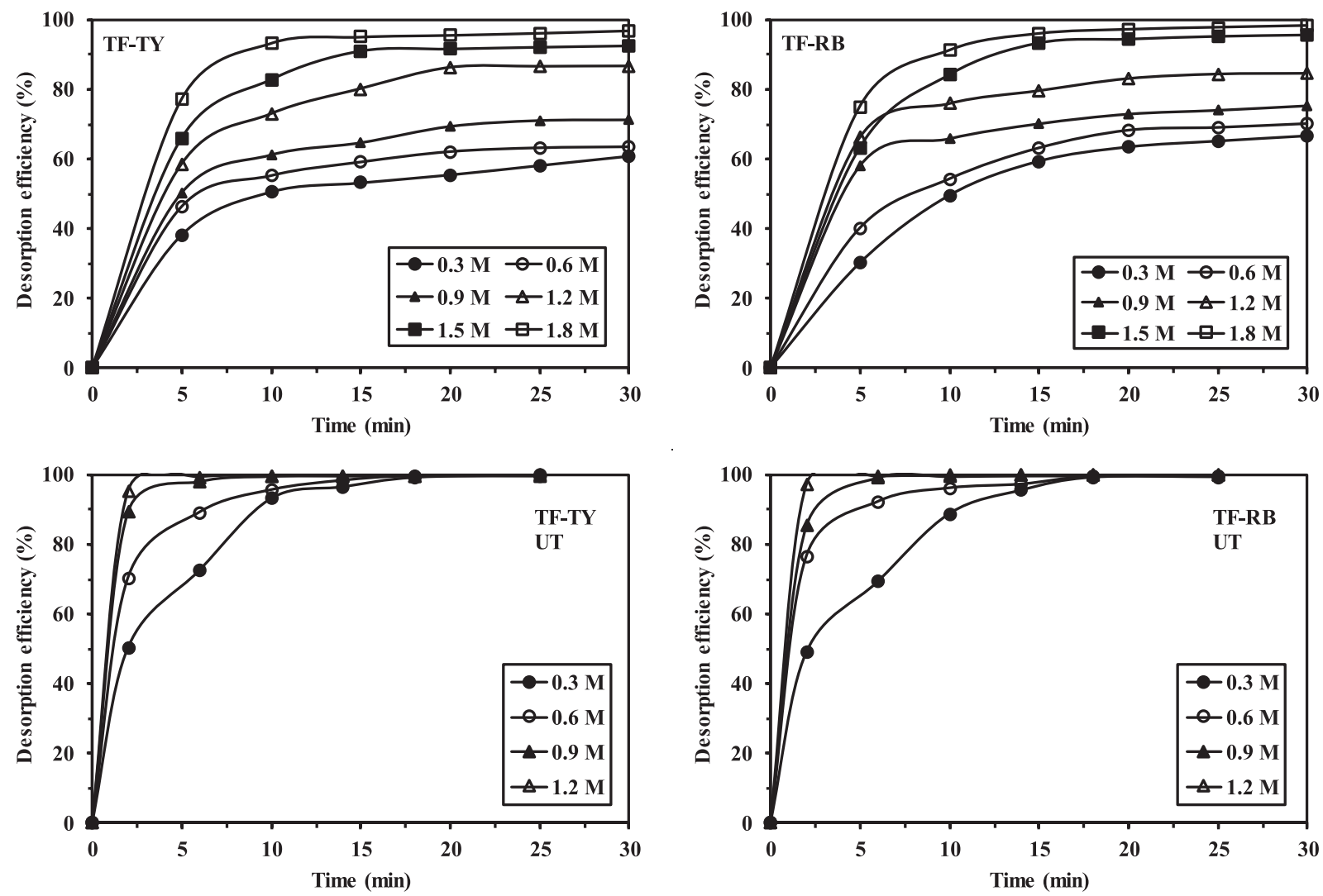

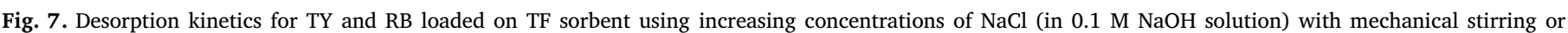

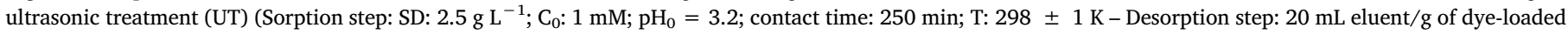
sorbent; T: $298 \pm 1 \mathrm{~K})$.

nitrate $(179)<$ chloride $(181)<$ hydrogen sulfate $(190)<$ hydrogen phosphate (200) [66]. The competitor effect is probably influenced by the combination of size and charge effects, in addition to possible differences in their ability to affect dye conformation in solution.

\subsubsection{Dye desorption and recycling of sorbents}

Desorption of the solute from loaded resins is a key step in the design of a sorption process. In the case of dye desorption from TF and MTF resins, the study of $\mathrm{pH}$ effect on TY and RB binding has shown a considerable decrease in sorption properties when $\mathrm{pH}$ exceeds 8 . Alkaline solutions may represent a solution for eluting the dyes. Preliminary tests showed that, at moderate concentration of $\mathrm{NaOH}$, the release of the dye is poorly effective and that the addition of $\mathrm{NaCl}$ strongly improves the effectiveness of the process. Therefore, the influence of increasing concentrations of $\mathrm{NaCl}$ (in background $0.1 \mathrm{M}$ 

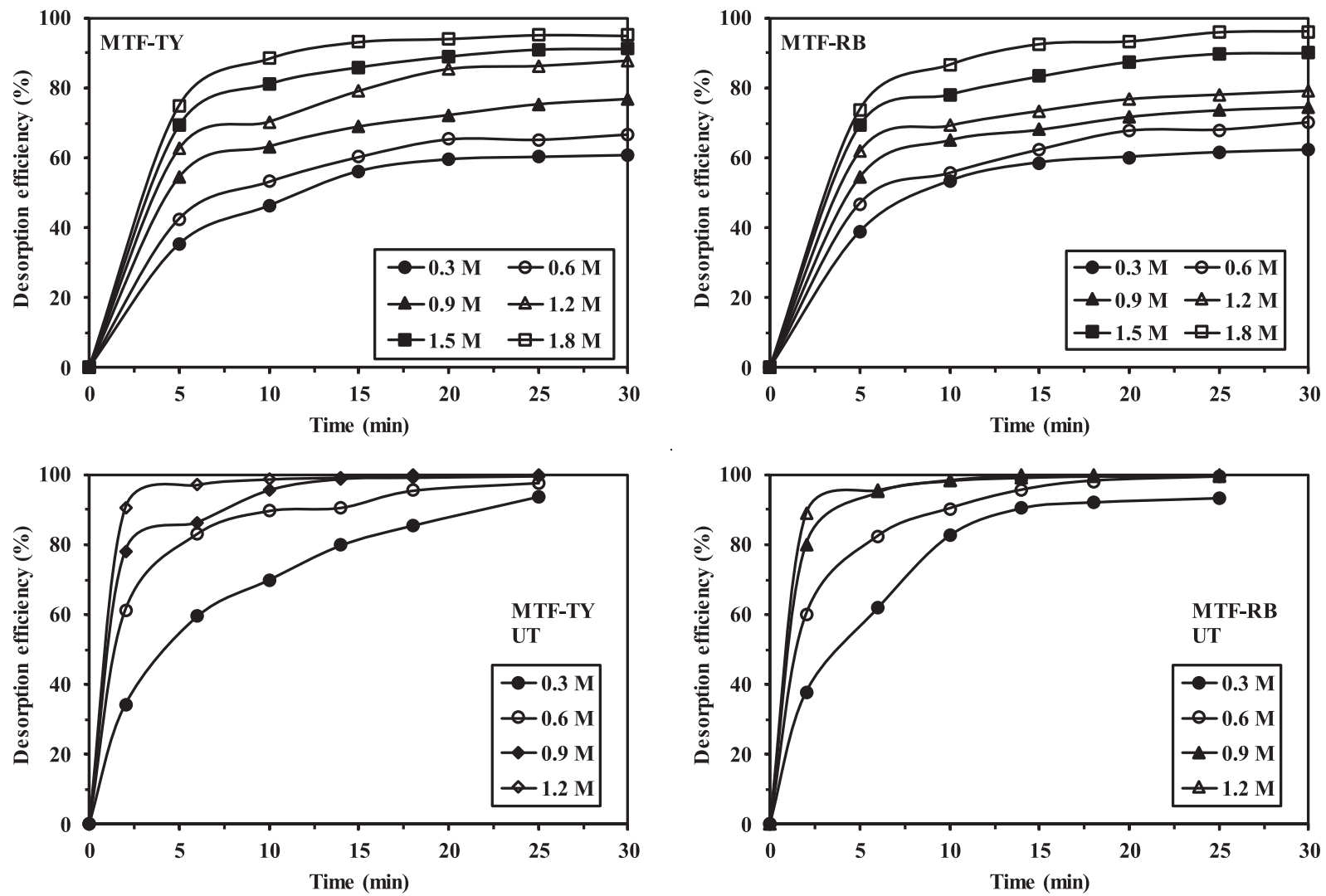

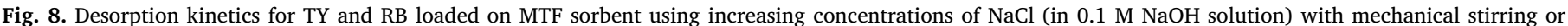

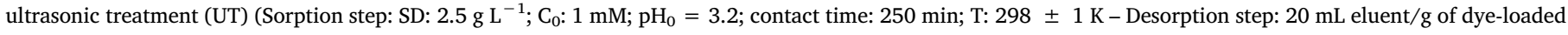
sorbent; T: $298 \pm 1 \mathrm{~K})$.

Table 5

Sorbent recycling - Comparison of desorption efficiencies (DE, \%) for TF and MTF on both TY and RB dyes for five successive cycles.

\begin{tabular}{|c|c|c|c|c|c|}
\hline \multirow{3}{*}{ Cycle \# } & \multirow{3}{*}{ Step } & \multicolumn{4}{|c|}{$\begin{array}{l}\text { Sorption capacity (q, mmol } \mathrm{g}^{-1} \text { ) or Desorption } \\
\text { efficiency }(\%)\end{array}$} \\
\hline & & \multicolumn{2}{|l|}{$\mathrm{TF}$} & \multicolumn{2}{|l|}{ MTF } \\
\hline & & TY & $\mathrm{RB}$ & TY & $\mathrm{RB}$ \\
\hline \multirow[t]{2}{*}{1} & Sorption (q) & 0.951 & 0.935 & 0.937 & 0.891 \\
\hline & Desorption (\%) & 99.7 & 99.8 & 98.7 & 99.3 \\
\hline \multirow[t]{2}{*}{2} & Sorption (q) & 0.930 & 0.922 & 0.903 & 0.858 \\
\hline & Desorption (\%) & 99.2 & 98.5 & 98.6 & 98.2 \\
\hline \multirow[t]{2}{*}{3} & Sorption (q) & 0.906 & 0.914 & 0.896 & 0.853 \\
\hline & Desorption (\%) & 98.1 & 98.2 & 97.3 & 96.8 \\
\hline \multirow[t]{2}{*}{4} & Sorption (q) & 0.911 & 0.899 & 0.894 & 0.835 \\
\hline & Desorption (\%) & 97.5 & 98.1 & 96.2 & 96.3 \\
\hline \multirow[t]{2}{*}{5} & Sorption (q) & 0.902 & 0.880 & 0.892 & 0.817 \\
\hline & Desorption (\%) & 97.1 & 96.5 & 96.0 & 95.3 \\
\hline
\end{tabular}

Experimental Conditions - Sorption step: SD: $2.5 \mathrm{~g} \mathrm{~L}^{-1}$; $\mathrm{C}_{0}: 1 \mathrm{mM}, \mathrm{pH}_{0}: 3.2$; contact time: $25 \mathrm{~min}$; T: $298 \pm 1 \mathrm{~K}$; ultrasonic agitation: frequency $35 \mathrm{kHz}$ and power $300 \mathrm{~W} /$ Desorption using $0.9 \mathrm{M} \mathrm{NaCl} / 0.1 \mathrm{M} \mathrm{NaOH}$ solution: $20 \mathrm{~mL} \mathrm{~g}^{-1}$ (dye-loaded sorbent), contact time: 15 min under ultrasonic agitation ( $35 \mathrm{kHz}$ ); T: $298 \pm 1 \mathrm{~K})$

$\mathrm{NaOH}$ solutions) was tested for the two dyes, and the two sorbents, using either mechanical agitation or ultrasonic treatments. The kinetic profiles have been compared since the concentration may play on both equilibrium desorption and mass transfer. Figs. 7 and 8 illustrate these desorption kinetics. The two sorbents exhibit very similar trends. The variation of experimental parameters shows common trends:

(a) under mechanical agitation, high concentrations of $\mathrm{NaCl}$ are required (higher than $1.5 \mathrm{M}$ ) and the contact time should be extended to 25-30 min to achieve a desorption higher than $95 \%$. Generally, the levels of desorption are slightly lower for MTF than for TF. This is contrary to the trends followed for sorption. However, in terms of kinetic behavior, MTF resins are little slower (consistently with uptake kinetics) compared to TF.

(b) under ultrasonic treatment, the desorption kinetics are much faster and require lower concentrations of $\mathrm{NaCl}$. Indeed, with $\mathrm{NaCl}$ concentrations as low as $0.3 \mathrm{M}$ for TF and as low as $0.6 \mathrm{M}$ for MTF it is possible to reach complete release of TY and RB within 20-25 min of contact. With $1.2 \mathrm{M} \mathrm{NaCl}$ solutions, the complete removal of the dyes is achieved within 3 min of contact for TF, while the contact time should be extended to $10-15$ min with MTF.

These results support the beneficial effect of ultrasonic treatment already observed on uptake kinetics in terms of desorption masstransfer properties: the cavitation effect enhances the action of $\mathrm{NaCl}$ in promoting the ion-exchange of chloride ions with anionic dyes within the sorbents.

Since the uptake kinetics obey a pseudo-second order rate equation, the corresponding equation for desorption step has been tested for modeling the kinetics of desorption [67]:

PSORE for desorption: $\frac{q(t)}{q_{0}}=\frac{1}{\beta_{2}+k_{d e s, 2} t}$

with: $\mathrm{q}_{0}\left(\mathrm{mmol} \mathrm{g}^{-1}\right)$ is the initial dye load on the resin, $\beta_{2}$ a parameter of the PSORE for desorption and $\mathrm{k}_{\mathrm{des}, 2}\left(\mathrm{~min}^{-1}\right)$ is the apparent rate constant for desorption based on PSORE. Figs. AM20 and AM21 show the time-plots for $\mathrm{q}(\mathrm{t}) / \mathrm{q}_{0}$ for the different systems (sorbent/dye/agitation mode) at increasing concentrations of $\mathrm{NaCl}$. The solid curves represent the fits of experimental data with the PSORE (Eq. 5) and the parameters reported in Table AM5. The fits became better with 
increasing $\mathrm{NaCl}$ concentration but the model roughly fits experimental profiles. The comparison of apparent rate constants for desorption (i.e., $\mathrm{k}_{\mathrm{des}, 2}$ ) confirms the conclusions raised from Figs. 7 and 8:

(i) The rate constant increases with $\mathrm{NaCl}$ concentration (from 8 to 12 times in the case of mechanical agitation and from 11 to 36 times under ultrasonic treatment),

(ii) The rate constants are of the same order of magnitude for TY and $\mathrm{RB}$

(iii) The ultrasonic treatment considerably increases the rate constant compared with MA (from 10 to 50 times, depending on the system)

(iv) The rate constants are generally lower for MTF than for TF: the ultrasonic treatment increases the difference between the two sorbents (compared to simple mechanical agitations).

Table 5 compares for the two sorbents and the two dyes the recycling performances (sorption capacities and desorption efficiencies) for five successive cycles of sorption and desorption. The sorption properties are remarkably stable: at the fifth cycle, the sorption capacity is reduced by less than $6-8 \%$; the most important decrease (i.e., $8.3 \%$ ) is observed for the system: MTF/RB. This means that despite the repeated use of ultrasonic treatment the sorbents maintain a good physical-chemical stability. The desorption efficiency remains remarkably stable; despite a progressive decrease, at the fifth cycle the loss in desorption yield does not exceed $4 \%$. The sorbents present good recycling properties; making these materials promising for this kind of application.

\section{Conclusion}

The incorporation of magnetite particles into thiourea-formaldehyde resin (TF) allows producing a magnetic sorbent (MTF) with enhanced sorption properties, despite the relative decrease in the amount of reactive groups (the magnetite represents about $23 \%$ of total weight).

FTIR and NMR analysis clearly confirm the presence of expected reactive groups associated to $\mathrm{N}$ and $\mathrm{S}$ elements. The analysis of zeta potential confirms that the sorbents have $\mathrm{pH}_{\mathrm{PZC}}$ values in the range 6.1-6.9. This makes the sorbents protonated in acidic solutions, and available for the binding of anionic dyes such as titan yellow (bearing sulfonic groups) and rose bengal (bearing carboxylate groups) through an ion-exchange mechanism. The optimum initial $\mathrm{pH}$ is around 3; however, after dye sorption, the $\mathrm{pH}$ tends to stabilize around 5.2-5.8. The optimum $\mathrm{pH}$ results from (a) charge compatibility between the sorbents and the functional groups of the dyes, and (b) controlled competition of competitor ions (dissociation of acid and counter anions at low $\mathrm{pH}$ ). The incorporation of magnetite (though decreasing the effective amount of polymer) improves the sorption performance (essentially maximum sorption capacity but little negative effect on uptake kinetics); at least under mechanical agitation. Indeed, in the case of ultrasonic treatment, the cavitation effects not only improve the mass transfer performance but also the availability/accessibility of reactive groups. The ultrasonic treatment minimizes the differences between the two sorbents. The uptake kinetics are modeled using the pseudo-second order rate equation; although the resistance to intraparticle diffusion plays a role in the control of mass transfer (good fit of kinetic profiles with the Crank equation). Playing with the velocity of the agitation confirms that at low agitation speed, the resistance to film diffusion cannot be neglected. The ultrasonic treatment improves significantly dye mass transfer: equilibrium time is reduced from 180-210 min (MA) to $30 \mathrm{~min}$. This beneficial effect is also observed during the desorption of the dyes using alkaline $\mathrm{NaCl}$ solutions $(0.1 \mathrm{M} \mathrm{NaOH}$ solutions completed with increasing concentrations of $\mathrm{NaCl}$ ). The sorbents can be recycled for at least five cycles with limited decrease in sorption performances. The ultrasonic treatment speeds up dye desorption but also decreases the required amount of $\mathrm{NaCl}$, compared with mechanical agitation. Kinetic profiles of desorption are effectively fitted by the pseudo-second order rate equation. Ultrasonic treatment appears very effective for process intensification (both in terms of sorption and desorption).

\section{Declaration of Competing Interest}

The authors declare that they have no known competing financial interests or personal relationships that could have appeared to influence the work reported in this paper.

\section{Acknowledgements}

This work was funded by the University of Jeddah (Saudi Arabia), under grant No. UJ-39-18-DR. The authors, therefore, acknowledge with thanks the University technical and financial support. Also the authors acknowledge Institut Français d'Egypte for supporting the binational collaboration France-Egypt.

\section{Appendix A. Supplementary data}

Supplementary data to this article can be found online at https:// doi.org/10.1016/j.cej.2019.123635.

\section{References}

[1] N. Zhuo, Y. Lan, W. Yang, Z. Yang, X. Li, X. Zhou, Y. Liu, J. Shen, X. Zhang, Adsorption of three selected pharmaceuticals and personal care products (PPCPs) onto MIL-101(Cr)/natural polymer composite beads, Sep. Purif. Technol. 177 (2017) 272-280.

[2] X. Wang, N. Zhuo, C. Fu, Z. Tian, H. Li, J. Zhang, W. Wu, Z. Yang, W. Yang, Enhanced selective adsorption of benzotriazole onto nanosized zeolitic imidazolate frameworks confined in polystyrene anion exchanger, Chem. Eng. J. 328 (2017) $816-824$.

[3] Y. Tang, Z. Liu, K. Zhao, S. Fu, Adsorption and separation properties of positively charged $\mathrm{ZrO}_{2}$ nanofibrous membranes fabricated by electrospinning, RSC Adv. 7 (2017) 42505-42512.

[4] X. Cheng, N. Li, M. Zhu, L. Zhang, Y. Deng, C. Deng, Positively charged microporous ceramic membrane for the removal of Titan Yellow through electrostatic adsorption, J. Environ. Sci. 44 (2016) 204-212.

[5] S.D. Khattri, M.K. Singh, Removal of malachite green from dye wastewater using neem sawdust by adsorption, J. Hazard. Mater. 167 (2009) 1089-1094.

[6] A. Mittal, D. Kaur, J. Mittal, Batch and bulk removal of a triarylmethane dye, Fast Green FCF, from wastewater by adsorption over waste materials, J. Hazard. Mater. 163 (2009) 568-577.

[7] K.T. Chung, Mutagenicity and carcinogenicity of aromatic amines metabolically produced from azo dyes, J. Environ. Sci. Health., Part C 18 (2000) 51-74.

[8] M.A. Rauf, S.S. Ashraf, Survey of recent trends in biochemically assisted degradation of dyes, Chem. Eng. J. 209 (2012) 520-530.

[9] A. Szygula, E. Guibal, M.A. Palacin, M. Ruiza, A.M. Sastre, Removal of an anionic dye (Acid Blue 92) by coagulation-flocculation using chitosan, J. Environ. Manage. 90 (2009) 2979-2986.

[10] H. Zazou, H. Afanga, S. Akhouairi, H. Ouchtak, A.A. Addi, R.A. Akbour, A. Assabbane, J. Douch, A. Elmchaouri, J. Duplay, et al., Treatment of textile in dustry wastewater by electrocoagulation coupled with electrochemical advanced oxidation process, J. Water Process Eng. 28 (2019) 214-221.

[11] K.Z. Elwakeel, Removal of Reactive Black 5 from aqueous solutions using magnetic chitosan resins, J. Hazard. Mater. 167 (2009) 383-392.

[12] E. Kamaraj, S. Somasundaram, K. Balasubramani, M.P. Eswaran, R. Muthuramalingam, S. Parka, Facile fabrication of $\mathrm{CuO}-\mathrm{Pb}_{2} \mathrm{O}_{3}$ nanophotocatalyst for efficient degradation of Rose Bengal dye under visible light irradiation, Appl. Surf. Sci. 433 (2018) 206-212.

[13] A.A. Tashvigh, L. Luo, T.-S. Chung, M. Weber, C. Maletzko, A novel ionically crosslinked sulfonated polyphenylsulfone (SPPSU) membrane for organic solvent nanofiltration (OSN), J. Membr. Sci. 545 (2018) 221-228.

[14] K.Z. Elwakeel, A.M. Elgarahy, S.H. Mohammad, Use of beach bivalve shells located at Port Said coast (Egypt) as a green approach for methylene blue removal, J. Environ. Chem. Eng. 5 (2017) 578-587.

[15] G. McKay, A. Mesdaghinia, S. Nasseri, M. Hadi, M.S. Aminabad, Optimum isotherms of dyes sorption by activated carbon: Fractional theoretical capacity \& error analysis, Chem. Eng. J. 251 (2014) 236-247.

[16] A. Balis, S. Zapotoczny, Tailored synthesis of core-shell mesoporous silica particlesoptimization of dye sorption properties, Nanomaterials 8 (2018).

[17] W.S.W. Ngah, L.C. Teong, M.A.K.M. Hanafiah, Adsorption of dyes and heavy metal ions by chitosan composites: a review, Carbohydr. Polym. 83 (2011) 1446-1456.

[18] Z. Zhou, S. Lin, T. Yue, T.-C. Lee, Adsorption of food dyes from aqueous solution by glutaraldehyde cross-linked magnetic chitosan nanoparticles, J. Food Eng. 126 (2014) 133-141. 
[19] E.C. Chigbundu, K.O. Adebowale, Equilibrium and fractal-like kinetic studies of the sorption of acid and basic dyes onto watermelon shell (Citrullus vulgaris), Cellulose 24 (2017) 4701-4714.

[20] X. Luo, Y. Zhan, Y. Huang, L. Yang, X. Tu, S. Luo, Removal of water-soluble acid dyes from water environment using a novel magnetic molecularly imprinted polymer, J. Hazard. Mater. 187 (2011) 274-282.

[21] Y. Shao, L. Zhou, C. Bao, J. Ma, M. Liu, F. Wang, Magnetic responsive metal-organic frameworks nanosphere with core-shell structure for highly efficient removal of methylene blue, Chem. Eng. J. 283 (2016) 1127-1136.

[22] J. Fei, Y. Cui, X. Yan, W. Qi, Y. Yang, K. Wang, Q. He, J. Li, Controlled preparation of $\mathrm{MnO}_{2}$ hierarchical hollow nanostructures and their application in water treatment, Adv. Mater. 20 (2008) 452-456.

[23] S. Wang, Z. Xiao, X. Ma, Z. Zhao, D. Guo, Y. Chen, S. Zhai, Q. An, D. Yang, Hard template-induced internal solidification synthesis of Cu NPs-supported glutaraldehyde-crosslinked polyethyleneimine-modified calcium alginate beads with enhanced catalytic activity, Appl. Catal., A 568 (2018) 105-113.

[24] S. Qadri, A. Ganoe, Y. Haik, Removal and recovery of acridine orange from solutions by use of magnetic nanoparticles, J. Hazard. Mater. 169 (2009) 318-323.

[25] V. Belessi, G. Romanos, N. Boukos, D. Lambropoulou, C. Trapalis, Removal of Reactive Red 195 from aqueous solutions by adsorption on the surface of $\mathrm{TiO}_{2}$ nanoparticles, J. Hazard. Mater. 170 (2009) 836-844.

[26] G. Moussavi, M. Mahmoudi, Removal of azo and anthraquinone reactive dyes from industrial wastewaters using $\mathrm{MgO}$ nanoparticles, J. Hazard. Mater. 168 (2009) 806-812.

[27] G. Absalan, M. Asadi, S. Kamran, L. Sheikhian, D.M. Goltz, Removal of reactive red120 and 4-(2-pyridylazo) resorcinol from aqueous samples by $\mathrm{Fe}_{3} \mathrm{O}_{4}$ magnetic nanoparticles using ionic liquid as modifier, J. Hazard. Mater. 192 (2011) 476-484.

[28] A. Asfaram, M. Ghaedi, S. Hajati, A. Goudarzi, E.A. Dil, Screening and optimization of highly effective ultrasound-assisted simultaneous adsorption of cationic dyes onto $\mathrm{Mn}$-doped $\mathrm{Fe}_{3} \mathrm{O}_{4}$-nanoparticle-loaded activated carbon, Ultrason. Sonochem. 34 (2017) 1-12.

[29] F. Ke, L.-G. Qiu, Y.-P. Yuan, X. Jiang, J.-F. Zhu, $\mathrm{Fe}_{3} \mathrm{O}_{4} @ M O F$ core-shell magnetic microspheres with a designable metal-organic framework shell, J. Mater. Chem. 22 (2012) 9497-9500.

[30] N. Gezer, M. Gulfen, A.O. Aydin, Adsorption of selenite and selenate ions onto thiourea-formaldehyde resin, J. Appl. Polym. Sci. 122 (2011) 1134-1141.

[31] Y. Zhao, L. Xu, M. Liu, Z. Duan, H. Wang, Magnetic mesoporous thiourea-formaldehyde resin as selective adsorbent: A simple and highly-sensitive electroanalysis strategy for lead ions in drinking water and milk by solid state-based anodic stripping, Food Chem. 239 (2018) 40-47.

[32] C.H. Ni, C.H. Yi, Z.Y. Feng, Studies of syntheses and adsorption properties of chelating resin from thiourea and formaldehyde, J. Appl. Polym. Sci. 82 (2001) 3127-3132.

[33] S.M. Alshehri, A. Al-Fawaz, T. Ahamad, Thermal kinetic parameters and evolved gas analysis (TG-FTIR-MS) for thiourea-formaldehyde based polymer metal complexes, J. Anal. Appl. Pyrol. 101 (2013) 215-221.

[34] N. Muslu, M. Gulfen, Selective separation and concentration of Pd(II) from Fe(III), $\mathrm{Co}(\mathrm{II}), \mathrm{Ni}(\mathrm{II})$, and $\mathrm{Cu}(\mathrm{II})$ ions using thiourea-formaldehyde resin, J. Appl. Polym. Sci. 120 (2011) 3316-3324.

[35] H.A. Panahi, A.A.M. Sharif, M. Bigonah, E. Moniri, Preconcentration and determination of chromium in water with flame atomic absorption spectrometry by thiourea-formaldehyde as chelating resin, Korean J. Chem. Eng. 26 (2009) 1723-1728.

[36] S. Kirci, M. Guelfen, A.O. Aydin, Separation and recovery of silver(I) ions from base metal ions by thiourea- or urea-formaldehyde chelating resin, Sep. Sci. Technol. 44 (2009) 1869-1883.

[37] E. Ertan, M. Guelfen, Separation of gold(III) ions from copper(II) and zinc(II) ions using thiourea-formaldehyde or urea-formaldehyde chelating resins, J. Appl. Polym. Sci. 111 (2009) 2798-2805.

[38] R. Oza, N. Shah, S. Patel, Recovery of nickel from spent catalysts using ultrasonication-assisted leaching, J. Chem. Technol. Biotechnol. 86 (2011) 1276-1281.

[39] P. Zhang, Y. Ma, F. Xie, Impacts of ultrasound on selective leaching recovery of heavy metals from metal-containing waste sludge, J. Mater. Cycles Waste Manage. 15 (2013) 530-538.

[40] O. Hamdaoui, M. Chiha, E. Naffrechoux, Ultrasound-assisted removal of malachite green from aqueous solution by dead pine needles, Ultrason. Sonochem. 15 (2008) 799-807.

[41] M. Roosta, M. Ghaedi, R. Sahraei, M.K. Purkait, Ultrasonic assisted removal of sunset yellow from aqueous solution by zinc hydroxide nanoparticle loaded activated carbon: Optimized experimental design, Mater. Sci. Eng., C 52 (2015) 82-89.

[42] S. Bagheri, H. Aghaei, M. Ghaedi, A. Asfaram, M. Monajemi, A.A. Bazrafshan, Synthesis of nanocomposites of iron oxide/gold $\left(\mathrm{Fe}_{3} \mathrm{O}_{4} / \mathrm{Au}\right)$ loaded on activated carbon and their application in water treatment by using sonochemistry:
Optimization study, Ultrason. Sonochem. 41 (2018) 279-287.

[43] T. Taghipour, G. Karimipour, M. Ghaedi, A. Asfaram, Mild synthesis of a Zn(II) metal organic polymer and its hybrid with activated carbon: Application as antibacterial agent and in water treatment by using sonochemistry: Optimization, kinetic and isotherm study, Ultrason. Sonochem. 41 (2018) 389-396.

[44] M. Dastkhoon, M. Ghaedi, A. Asfaram, R. Jannesar, F. Sadeghfar, Magnetic based nanocomposite sorbent combination with ultrasound assisted for solid-phase microextraction of Azure II in water samples prior to its determination spectrophotometric, J. Colloid Interface Sci. 513 (2018) 240-250.

[45] L. Lupa, R. Voda, A. Popa, Adsorption behavior of cesium and strontium onto chitosan impregnated with ionic liquid, Sep. Sci. Technol. 53 (2018) 1107-1115.

[46] K.Z. Elwakeel, A.A. El-Bindary, A.Z. El-Sonbati, A.R. Hawas, Adsorption of toxic acidic dye from aqueous solution onto diethylenetriamine functionalized magnetic glycidyl methacrylate-N,N'-methylenebisacrylamide, RSC Adv. 6 (2016) 3350-3361.

[47] R. Massart, Preparation of aqueous magnetic liquids in alkaline and acidic media, IEEE Trans. Magn. 17 (1981) 1247-1249.

[48] B. Zhang, J.M. Xing, H.Z. Liu, Synthesis and characterization of superparamagnetic poly(urea-formaldehyde) adsorbents and their use for adsorption of flavonoids from Glycyrrhiza uralensis Fisch, Adsorption - J. Int. Ads. Soc. 14 (2008) 65-72.

[49] M.F. Zawrah, E.S.E. El Shereefy, A.Y. Khudir, Reverse precipitation synthesis of $\leq 10 \mathrm{~nm}$ magnetite nanoparticles and their application for removal of heavy metals from water, Silicon 11 (2019) 85-104.

[50] T. Ahamad, S.M. Alshehri, Thermal degradation and evolved gas analysis of thiourea-formaldehyde resin (TFR) during pyrolysis and combustion, J. Therm. Anal. Calorim. 109 (2012) 1039-1047.

[51] M. Stoia, R. Istratie, C. Păcurariu, Investigation of magnetite nanoparticles stability in air by thermal analysis and FTIR spectroscopy, J. Therm. Anal. Calorim. 125 (2016) 1185-1198.

[52] K. Petcharoen, A. Sirivat, Synthesis and characterization of magnetite nanoparticles via the chemical co-precipitation method, Mater. Sci. Eng., B 177 (2012) 421-427.

[53] A.A. El-Bindary, A.F. Shoair, H.A. Kiwaan, A.R. Hawas, Preparation, characterization, and application of synthesized thiourea formaldehyde-calcium alginate in removal of Reactive Black 5, Can. J. Chem. 96 (2018) 1101-1114.

[54] M. Karunakaran, C.T. Vijayakumar, D. Muthamil Selvan, C. Magesh, o-Cresol, thiourea and formaldehyde terpolymer - A cation exchange resin, J. Saudi Chem. Soc. 17 (2013) 1-8.

[55] T. Ahamad, V. Kumar, N. Nishat, Synthesis, characterization and antimicrobial activity of transition metal chelated thiourea-formaldehyde resin, Polym. Int. 55 (2006) 1398-1406.

[56] M. Breitbach, D. Bathen, Influence of ultrasound on adsorption processes, Ultrason. Sonochem. 8 (2001) 277-283.

[57] A. Mary Ealias, M.P. Saravanakumar, A critical review on ultrasonic-assisted dye adsorption: Mass transfer, half-life and half-capacity concentration approach with future industrial perspectives, Crit. Rev. Environ. Sci. Technol. 49 (2019) 1959-2015.

[58] V.R. Batistela, D.S. Pellosi, F.D. de Souza, W.F. da Costa, S.M. de Oliveira Santin, V.R. de Souza, W. Caetano, H.P.M. de Oliveira, I.S. Scarminio, N. Hioka, pKa determinations of xanthene derivates in aqueous solutions by multivariate analysis applied to UV-Vis spectrophotometric data, Spectrochim. Acta, Part A 79 (2011) 889-897.

[59] N. Bao, Y. Li, X.H. Yu, J.J. Niu, G.L. Wu, X.H. Xu, Removal of anionic azo dye from aqueous solution via an adsorption-photosensitized regeneration process on $\mathrm{a} \mathrm{TiO}_{2}$ surface, Environ. Sci. Pollut. Res. 20 (2013) 897-906.

[60] S. Azizian, Kinetic models of sorption: a theoretical analysis, J. Colloid Interface Sci. 276 (2004) 47-52.

[61] S. Sarkar, M. Sarkar, Ultrasound assisted batch operation for the adsorption of hexavalent chromium onto engineered nanobiocomposite, Heliyon, 5 (2019) e01491-01420.

[62] M.I. Temkin, V. Pyzhev, Kinetics of ammonia synthesis on promoted iron catalysts, Acta Physiochim. 12 (1940) 217-222.

[63] S. Karcher, A. Kornmüller, M. Jekel, Anion exchange resins for removal of reactive dyes from textile wastewaters, Water Res. 36 (2002) 4717-4724.

[64] Y. Zhang, C. Zhu, F. Liu, Y. Yuan, H. Wu, A. Li, Effects of ionic strength on removal of toxic pollutants from aqueous media with multifarious adsorbents: A review, Sci. Total Environ. 646 (2019) 265-279.

[65] N.M. Mahmoodi, B. Hayati, M. Arami, C. Lan, Adsorption of textile dyes on Pine Cone from colored wastewater: kinetic, equilibrium and thermodynamic studies, Desalination 268 (2011) 117-125.

[66] Y. Marcus, Ion Properties, Marcel Dekker Inc., New York, NY, 1997, p. 259.

[67] N.K. Lazaridis, T.A. Pandi, K.A. Matis, Chromium(VI) removal from aqueous solutions by $\mathrm{Mg}-\mathrm{Al}-\mathrm{CO}_{3}$ hydrotalcite: sorption-desorption kinetic and equilibrium studies, Ind. Eng. Chem. Res. 43 (2004) 2209-2215. 